\title{
Multi-scale characterization of symbiont diversity in the pea aphid complex through metagenomic approaches
}

Cervin Guyomar ${ }^{1,2}$, Fabrice Legeai ${ }^{1,2}$, Emmanuelle Jousselin $^{3}$, Christophe Mougel ${ }^{1}$, Claire Lemaitre ${ }^{2}$ and Jean-Christophe Simon ${ }^{1 *}$

\begin{abstract}
Background: Most metazoans are involved in durable relationships with microbes which can take several forms, from mutualism to parasitism. The advances of NGS technologies and bioinformatics tools have opened opportunities to shed light on the diversity of microbial communities and to give some insights into the functions they perform in a broad array of hosts. The pea aphid is a model system for the study of insect-bacteria symbiosis. It is organized in a complex of biotypes, each adapted to specific host plants. It harbors both an obligatory symbiont supplying key nutrients and several facultative symbionts bringing additional functions to the host, such as protection against biotic and abiotic stresses. However, little is known on how the symbiont genomic diversity is structured at different scales: across host biotypes, among individuals of the same biotype, or within individual aphids, which limits our understanding on how these multi-partner symbioses evolve and interact.
\end{abstract}

Results: We present a framework well adapted to the study of genomic diversity and evolutionary dynamics of the pea aphid holobiont from metagenomic read sets, based on mapping to reference genomes and whole genome variant calling. Our results revealed that the pea aphid microbiota is dominated by a few heritable bacterial symbionts reported in earlier works, with no discovery of new microbial associates. However, we detected a large and heterogeneous genotypic diversity associated with the different symbionts of the pea aphid. Partitioning analysis showed that this fine resolution diversity is distributed across the three considered scales. Phylogenetic analyses highlighted frequent horizontal transfers of facultative symbionts between host lineages, indicative of flexible associations between the pea aphid and its microbiota. However, the evolutionary dynamics of symbiotic associations strongly varied depending on the symbiont, reflecting different histories and possible constraints. In addition, at the intra-host scale, we showed that different symbiont strains may coexist inside the same aphid host.

Conclusions: We present a methodological framework for the detailed analysis of NGS data from microbial communities of moderate complexity and gave major insights into the extent of diversity in pea aphid-symbiont associations and the range of evolutionary trajectories they could take.

Keywords: Host-microbiota interactions, Aphids, Metagenomics, Symbiosis, Phylogeny

\footnotetext{
* Correspondence: jean-christophe.simon@inra.fr

${ }^{1}$ INRA, UMR 1349 INRA/Agrocampus Ouest/Université Rennes 1, Institut de

Génétique, Environnement et Protection des Plantes (IGEPP), Le Rheu, France

Full list of author information is available at the end of the article
}

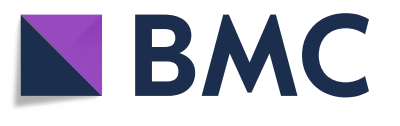

(c) The Author(s). 2018 Open Access This article is distributed under the terms of the Creative Commons Attribution 4.0 International License (http://creativecommons.org/licenses/by/4.0/), which permits unrestricted use, distribution, and

reproduction in any medium, provided you give appropriate credit to the original author(s) and the source, provide a link to the Creative Commons license, and indicate if changes were made. The Creative Commons Public Domain Dedication waiver (http://creativecommons.org/publicdomain/zero/1.0/) applies to the data made available in this article, unless otherwise stated. 


\section{Background}

Symbioses have been studied for long in the case of simple binary interactions between a host and a single symbiont. Many studies have unveiled the functional impacts and the evolutionary consequences of these symbioses including acquisition of novel functions, transmission patterns [1, 2], genomic changes [3], reproductive manipulations (reviewed in [4]), or cost/benefit balance of symbiotic relationships [5, 6]. Yet, the advances of molecular techniques in the last decades have revolutionized the description and understanding of host-microbe interactions and revealed that every plant or animal is interacting in some way with multiple microbes [7]. Biology is undergoing a paradigm shift where individual phenotypes should be considered as resulting from the combined expression of the host and associated microbe genomes (metagenomes) [8]. As a reflection of this conceptual shift, the term "holobiont" is now used to name the complex ecosystem of a host and its community of associated organisms [9, 10]. Similarly, the term "hologenome" is used to describe the collection of genomes of a host and its microbiota [11]. A prerequisite to understand the functional, ecological, and evolutionary implications of host-microbiota associations for holobionts is to evaluate the extent and partitioning of diversity at different scales involving individuals and populations of holobionts. This can be obtained from (i) a full inventory of the microbial entities associated with the host, including transient low abundant symbionts and (ii) a fine characterization of the genomic diversity of microbial partners both within and between individual hosts from different populations. Inter-individual host diversity is often ignored when pooling together several individuals, or underestimated by insufficient sampling in the population, and intra-host variability is rarely considered, but these two levels are essential to infer the evolutionary dynamics of host microbiota interactions [12] and to better link microbiota diversity with associated phenotypic changes in the host [13].

Next generation sequencing techniques can provide whole genome sequencing data of communities of organisms. Some host sequencing projects contain microbe-related reads that are often considered as "contaminant" in the analysis of the host genome. These datasets can actually be analyzed and provide meaningful insights about organisms seen as holobionts. Shotgun metagenomic sequencing has several features which enables high-resolution analysis of taxonomic and genetic diversity associated with holobionts. First, because it is a without a priori technique, it can capture all of the microbial diversity in environmental or host samples, including unknown bacteria, viruses, or eukaryotic symbionts. Secondly, it provides whole genome information, which enables to detect genetic variation at a fine scale and therefore offers the potential to track the evolutionary history of the holobiont partners, including acquisition source and gain-loss dynamics of microbial diversity. One criticism on metagenomic studies investigating the genetic diversity associated with holobionts is that most of the current phylogenetic analyses using the bacterial 16S ribosomal RNA gene are led at a coarse scale. They cannot assess accurately the specificity of the association between a host and its symbionts because bacteria with similar 16S rRNA (usually above 97\% sequence identity) can have substantial differences on the rest of the genome and therefore have different impacts on their host phenotypes [14]. Whole genome metagenomic sequencing allows investigating fine-scale diversity and yields robust phylogenetic information. Moreover, the whole genome information can be used to explore the phenotypic effects of symbiotic communities by using gene annotations and reconstructing holobiont metabolic networks [15].

Over the last decades, numerous computational methods have been developed to improve the analysis of metagenomic reads. These bioinformatics developments can be grouped into two main approaches: de novo genome assembly and metagenomic sequence profiling that is the grouping of sequences from one or several metagenomes into groups of the same taxonomical origin. Both of these approaches have been mainly applied to examine diversity at the species-level. If tremendous progress has been achieved in de novo metagenomics assembly [16], the inherent goal remains to build a set of consensus sequences representing the actual species in the metagenomics sample and polymorphism information is usually discarded, preventing the recovery of strain-level genomic variations $[17,18]$. On the other hand, metagenomic profiling when based on reference databases is either restricted to few marker genes $[19,20]$ or can perform strain-level assignation only for model systems or very well studied organisms for which many strains are already characterized (for instance for biomedically important pathogens [21, 22]). Finally, reference-free metagenomic profiling approaches, also called binning approaches, are often based on previous assemblies that have already discarded polymorphism information [23, 24] or, when using co-abundance signals, may lead to incorrect binning when conserved and variable regions of a same species are sorted in different bins [25].

Overall, one of the main pitfalls of current holobiont analyses is the characterization of microbes at strain/genotype level. Apart from model communities for which comprehensive strain databases are available, fine variations in symbiont genomes are not accurately addressed by the current metagenomics-dedicated methods.

Then, a basic but efficient strategy consists in converting the problem into several non-metagenomic ones, 
namely analyzing each symbiont and its corresponding read subsets independently using classical genomic variation methods. The major difficulty remains to be able to partition unambiguously the read datasets, and this is definitely easier when disposing of good reference genomes for all the symbionts.

In the present paper, we present a framework designed to recover strain-level genomic variations from metagenomic reads preliminary mapped on reference genomes. When a given symbiont lacks a good reference genome, it is then built de novo from the metagenomic datasets.

To assess the potential value offered by this framework, we applied it to a biological system of moderate complexity regarding microbial communities and with good prior knowledge of the expected symbiotic diversity. The pea aphid Acyrthosiphon pisum is a model species for insect symbioses and shows several features which make it relevant for studying the factors structuring microbial diversity in holobionts. Pea aphids shelter an obligate bacterial symbiont, Buchnera aphidicola which provides the host with essential amino acids absent or scarce in the insect diet (i.e., phloem sap [26]). In addition, several secondary symbionts are commonly found in pea aphid populations at different frequencies. Some of these secondary symbionts have been shown to provide ecological advantages to their hosts, for example, by increasing protection against natural enemies or by conferring thermal tolerance [27]. While the primary symbiont is strictly maternally inherited [28], secondary symbionts are vertically transmitted with a lower fidelity and can be horizontally transmitted [29], but neither the mechanisms nor the magnitude of these events of horizontal transfers are fully understood [30]. The pea aphid actually forms a complex of at least 15 biotypes, each biotype being adapted to a specific set of host plants [31]. Estimates of divergence time between biotypes suggest that this complex may have diversified 5000-10,000 years ago, which coincides with the onset of plant domestication for agriculture [32, 33]. Population genetic analyses revealed that these biotypes form a continuum of divergence, with partially isolated host races and reproductively isolated cryptic species [32]. Several studies revealed that pea aphid biotypes also differ in their composition and frequency of secondary symbionts, but secondary symbionts seem to contribute very little to plant specialization of their hosts [31, 3436]. In addition, strain variation has been characterized in some secondary symbionts infecting the pea aphid complex [35] and found in some cases associated with large phenotypic differences in their hosts [37, 38]. Overall, the available literature on the pea aphid symbionts indicates large variation across host populations, both in bacterial species and strains, with important functional, ecological, and evolutionary impacts on pea aphid holobionts. Although there have been recent attempts to uncover the bacterial communities associated with the pea aphid complex with deep sequencing of $16 \mathrm{~S}$ ribosomal RNA [34, 39], no study has been yet conducted to fully characterize the diversity of pea aphid microbiota notably at different scales of organization and at a whole genome scale. The pea aphid appears to be a relevant system to develop a metagenomic framework applied to the analysis of microbial diversity and structure in holobionts. It is located at a sweet spot of complexity, with a symbiotic community of moderate size and with various modes of transmission of symbionts between hosts. It offers an interesting case of diversity partitioning between host populations through genetically and ecologically differentiated biotypes, and it is a species for which ample genomic resources are available for both the host and its associated symbionts.

In this paper, we analyzed metagenomic data from a large dataset of pea aphid-resequenced genomes to explore the extent and partitioning of microbial diversity at the different scales presented above. By mapping the reads on a set of reference genomes, we assigned the majority of the reads to microbial taxa associated with the pea aphid complex. This enabled a high-resolution inventory of the genomic diversity of bacterial symbionts found in the pea aphid complex. Variant calling and phylogenetic approaches on the whole set of symbiotic bacteria revealed contrasted levels of genomic variability and various transmission patterns between symbionts, presumably resulting from different evolutionary histories and ecologies of host-symbiont associations.

\section{Methods}

\section{Biological samples}

Pea aphids were collected on different plants of the Fabaceae family mainly in eastern France where host plant diversity is high but also in southern and western France (Additional file 1). Individuals were sampled as parthenogenetic (clonal) females and brought to the laboratory to initiate individual clonal lineages. After at least two generations of culture on broad bean Vicia faba (a plant on which all pea aphid biotypes can feed [40]), DNA was extracted from each clone in order to (i) genotype them with several polymorphic microsatellite markers, (ii) detect repeated genotypes (i.e., individuals having the same multilocus genotypes and thus presumably belonging to the same clone) and remove them from further analyses to keep a single copy per genotype, and (iii) check biotype membership of each lineage through assignment tests (see [41] for further details). Briefly, individuals with a membership equal or larger than $90 \%$ in the genetic cluster corresponding to their assigned biotype were selected for further sequencing scheme. In this study, 14 biotypes out of the 15 
described for the pea aphid complex were each represented either by single or pooled individuals. Thirty-two individual resequenced genotypes encompassing 11 biotypes were those already used in [42]. This study also includes 18 new samples corresponding to pools of 14 to 35 individuals, each with a distinct multilocus genotype but belonging to the same biotype following assignment tests, representing overall 12 biotypes. Overall, the 50 samples used in this study are described in Additional file 1. Since these samples were composed of clones reared in the laboratory for at least two generations prior to DNA extraction for sequencing, their microbiota was largely composed of the heritable fraction, which was the focus of our study.

The DNA of the aphids and their microbiota was extracted using the Qiagen DNeasy Blood and Tissue Kit (Qiagen, Hilden, Germany) following the manufacturer's instructions and sequenced in paired end using Illumina HiSeq 2000 instruments, resulting in $2 \times 100$ bp reads with a mean insert size of $250 \mathrm{bp}$. The average read depth for the pea aphid genome was $15 \times$ for individual sequencing (42.5 million reads on average) and ranged from 20 to $50 \times$ for pool sequencing (197.5 million reads on average). FastQC files were generated for each sample, and no anomaly in the sequencing data was observed. The FastQ files of the paired reads from the 50 samples are stored and publicly available at the Sequence Read Archive of the National Center for Biotechnology Information database, under the BioProject IDs PRJNA255937, PRJNA385905, and PRJNA454786.

\section{Bioinformatics analyses}

Full details on the analysis presented in the following parts are available on the website https://aphid-microbiome.netlify.com. This includes the source code of every custom script used during the analyses.

\section{Mapping-based disentanglement of holobiont genomes}

Sequencing of both host and microbial DNA produces metagenomic datasets, containing reads originating from different organisms. This metagenomic context was dealt with by mapping read sets using BWA-MEM [43] with default parameters against a set of reference genomes, including the pea aphid nuclear and mitochondrial genomes, the primary symbiont genome (Buchnera aphidicola), and genomes of known pea aphid secondary symbionts, when available. This was the case for Hamiltonella defensa 5A, Serratia symbiotica Tucson, and Rickettsiella viridis and Regiella insecticola 5.15. For the Rickettsia symbiont, no closely related reference genome was available and we produced our own reference genome by de novo assembly, as explained in the paragraph below. For Spiroplasma, we used a draft genome previously assembled from unmapped reads of a particular pea aphid sample, as described in [42]. For Fukatsuia symbiotica (also named PAXS), we used the draft genome sequenced from the conifer aphid Cinara confinis $[44,45]$. In addition, we included in the reference set the variant genomes of the phage APSE of $H$. defensa [46] and several plasmid sequences associated to symbionts detected in the pea aphid. In particular, we added three Rickettsia plasmid sequences from other insects in order to map Rickettsia plasmidic reads in the absence of a reference sequence for A. pisum. After the mapping step, several statistics were computed, including the mapping rate, the average coverage for each genome, the fraction of the reference genome covered by at least five reads, and the mean edit distance for the reads mapping on each reference genome. Reads associated to each symbiont were extracted using Samtools [47], and all downstream analyses were conducted independently and with the same settings for each symbiont. The reference genomes used for this step are summarized in Table 1 . Additional statistics on the genomes used are available in Additional file 2.

\section{Assembly of Rickettsia sp. genome}

Using the results of a previous mapping of pea aphid reads on the genome of Rickettsia bellii, we identified two samples from the Pisum sativum biotype with high Rickettsia coverage (Ps_ind1 and Ps_ind2). These two samples were pooled together, resulting in a $100 \times$ coverage on the genome of $R$. bellii. Reads that mapped on the pea aphid genome were filtered out, and the remaining ones were assembled using SPAdes version 3.11.1 [48], with default parameters. Contigs with blast matches on Rickettsia bellii and Rickettsia sp MEAM1 were extracted. To increase contiguity and genome completeness, some pairs of contigs were bridged together using the gapfiller MindTheGap [49] that performs local assembly using the whole read set.

The resulting assembly was 1,070,000 bp long (for comparison, $R$. bellii is $1.5 \mathrm{Mb}$ long and Rickettsia sp. strain MEAM1 is $1.24 \mathrm{Mb}$ ), organized in 327 contigs, and had a N50 of 4483 bp. Eighty-two percent of complete genes were found using Busco v3.0.1 and the bacteria_odb9 gene set, which is very close to the $83.7 \%$ obtained for the reference genome of Rickettsia bellii. Compared to Rickettsia bellii, we observed a major improvement of the genome coverage as $84 \%$ more reads mapped on the newly assembled genome across the whole dataset.

\section{Analysis and taxonomic assignation of unmapped reads} Unmapped reads were extracted using Samtools [47], and low-quality reads were removed using Trimmomatic [50] with the following parameters: LEADING:3, TRAILING:3, SLIDINGWINDOW:4:15, and MINLEN:36. 
Table 1 Summary of reference genomes used for mapping

\begin{tabular}{|c|c|c|c|}
\hline Organism name & Sequence ID & Accession & Reference \\
\hline Acyrthosiphon pisum & Genome & SAMN00000061 & [85] \\
\hline \multirow[t]{3}{*}{ Buchnera aphidicola } & Genome APS & BA000003.2 & [86] \\
\hline & Plasmid pLeu & AP001071.1 & [86] \\
\hline & Plasmid pTrp & AP001070.1 & [86] \\
\hline \multirow[t]{8}{*}{ Hamiltonella defensa 5AT } & Genome 5AT & СР001277.1 & [87] \\
\hline & Plasmid pHD5AT & СР001278.1 & [87] \\
\hline & Phage APSE1 & AF157835.1 & [88] \\
\hline & Phage APSE3 & EU794053.1 & [46] \\
\hline & Phage APSE4 & EU794051.1 & [46] \\
\hline & Phage APSE5 & EU794050.1 & [46] \\
\hline & Phage APSE6 & EU794054.1 & [46] \\
\hline & Phage APSE7 & EU794052.1 & [46] \\
\hline \multirow[t]{2}{*}{ Regiella insecticola 5.15} & Genome & AGCA01000000 & [89] \\
\hline & Plasmid pRILSR1 & CM000957.1 & [90] \\
\hline Serratia symbiotica strain Tucson & Genome & GCA_000186485.2 & [91] \\
\hline Spiroplasma sp. & Genome & Upon request & [42] \\
\hline Fukatsuia symbiotica & Genome & GCA_900128755.1 & [44] \\
\hline Rickettsiella viridis & Genome & AP018005.1 & [92] \\
\hline \multirow[t]{4}{*}{ Rickettsia sp. } & Genome & Upon request & This paper \\
\hline & plasmid pREIS3 & CM000771.1 & \\
\hline & plasmid pRF & GQ329881.1 & \\
\hline & plasmid pRAF & СР001613.1 & \\
\hline Wolbachia sp. wRi & Genome & GCA_000022285.1 & [93] \\
\hline
\end{tabular}

Remaining unmapped reads were taxonomically assigned using Centrifuge [51]. Only assignation hits larger than 40 base pairs were kept. Results were visualized using the Pavian $\mathrm{R}$ package [52].

\section{Genome-wide variant calling}

Variant calling was performed for the whole set of symbionts identified in the pea aphid samples ( $B$. aphidicola, H. defensa, $R$. insecticola, S. symbiotica, Rickettsia sp., Spiroplasma sp., F. symbiotica, and $R$. viridis). It was also performed on the pea aphid mitochondrial genome, in order to capture the host matriline diversity. By essence, secondary symbionts were not present and equally abundant in all the samples, and a minimal coverage was required to run variant calling. Only symbionts with more than $10 \times$ sequencing depth and a homogeneous coverage along the genome were kept for this analysis. For instance, two symbionts in five samples were discarded because more than $90 \%$ of genomic positions were covered by less than two reads. This metric was smaller than $30 \%$ in the remaining samples.

Samtools mpileup [47] was used with options "-t DP,DPR" on the alignments to detect both SNPs and indels, and the coverage of the different alleles was reported. The generated bcf file was processed using bcftools [53] with options "-mv -Ov". Abundance tables of reference and alternative alleles for each polymorphic site and for each sample were extracted for further filtering using vcftools [54] and processed using a custom $\mathrm{R}$ script (available on the https://aphid-microbiome.netlify.com). In order to remove false positive variants due to sequencing errors, rare variants were removed by applying two coverage filters: for each sample, variants covered by less than four reads or with less than $10 \%$ frequency were removed. Regions with exceptionally high or low coverage were excluded from the analysis. Genomic positions were considered of low coverage when at least $75 \%$ of samples had a coverage inferior to the median coverage of all variants along the genome. Similarly, high-coverage genomic positions were discarded when the coverage was at least five times superior to the median coverage for at least $75 \%$ of the samples. In addition, for closely related reference genomes, such as $R$. insecticola, $H$. defensa, and F. symbiotica, homologous genomic regions were detected by performing a pairwise blast search, and regions with a homology greater than $80 \%$ were excluded. 


\section{Phylogenetic inference}

Variant frequencies were used to compute the variant profile of each sample by selecting the most abundant allele at each site. In the case of equally covered alleles, the reference allele was kept. This situation made it difficult to determine the most abundant genotype in the sample but was rare in our dataset. We therefore decided to remove from the analysis samples in which more than $5 \%$ of variable sites yielded alleles with equal abundances. It was the case for three pool sequencing samples with low symbiotic coverage.

To investigate the evolutionary relationships between the genomes of the different samples, a phylogenomic analysis on a set of gene encoding membrane proteins was performed when an annotated reference genome was available. We first selected a list of genes, in order to compute the putative sequences for these genes in all samples. The Uniprot database was queried to retrieve DNA sequences of membrane protein transcripts (under the "Cell membrane" keyword) for the different studied symbionts (the complete list of genes used can be found in Additional file 3). Membrane proteins were selected as they are assumed to show a higher mutation rate than usual phylogenetic markers [55] and therefore are more appropriate to capture recent phylogenetic events. This query resulted in sets of 96, 118, 141, and 96 genes for $B$. aphidicola, $H$. defensa, S. symbiotica, and R. insecticola, respectively. For each sample, the putative sequences of the selected proteins were inferred by replacing the reference alleles by the alternative alleles associated to the different variant profiles.

The gene sequences of each selected protein were aligned using MAFFT [56] (v7.310, linsi mode), and the resulting multiple alignments were concatenated. The lengths of the alignments for the analyzed symbionts were $92,293 \mathrm{bp}$ for $B$. aphidicola, 118,344 bp for $H$. defensa, 100,027 bp for $R$. insecticola, and 144,360 bp for S. symbiotica. To validate that our alignments were not subject to substitution saturation, a Xia's test was run, as implemented in DAMBE6 [57]. Because most software of phylogenetic inference struggle to estimate branch lengths for identical sequences, we pre-processed our concatenated alignments by keeping only one sequence for each set of identical sequences. We used RaxML [58] (version 8.2.10, options -f a -\# 1000 -m GTRGAMMA), a phylogenetic inference program based on maximum likelihood method, to infer the phylogeny of the samples of the considered genes. The GTRGAMMA model was used with no partitioning of the data matrix, with 1000 bootstrap iterations. Phylogenetic trees were edited and compared using functions of Ape [59] and Dendextend [60] R packages.

To cross-validate the phylogenetic relationships inferred from gene sets and also use the information contained in whole genome data, we used a clustering approach of whole genome variant profiles. Pairwise comparisons of variant profiles were performed; the numbers of differences between all pairs of profiles were then computed and divided by the total number of variants detected on the genome, as implemented in the AW-clust algorithm proposed in [61]. The distance matrix was then used to perform neighbor joining clustering and build a phylogenetic tree based on whole genome variant profile information. Tree topologies were visually compared between the gene set and whole genome approaches. For F. symbiotica, Rickettsia sp., R. viridis, and Spiroplasma sp., we did not perform a gene-based phylogeny since their reference genomes are not well assembled nor annotated. In that case, neighbor joining was performed on whole genome variant profiles to infer phylogenetic relationships between samples.

Outgroups were used to root the phylogenetic trees. For $B$. aphidicola, we used sequencing data of two Japanese $A$. pisum lineages, known to be highly divergent from European lineages [33]. For other symbionts, we used close-related symbiont species: $H$. defensa from the whitefly Bemisia tabaci (GenBank 2,777,848), S. symbiotica SCt-Vlc from the conifer aphid Cinara tujafilina (FR904230), Spiroplasma melliferum KC3 from Apis mellifera (GCA_000236085.3), Rickettsia sp. MEAM1 from Bemisia tabaci (GCA_002285905.1), and Rickettsiella grylli from crickets (GCA_000168295.1). For $R$. insecticola, the closest known symbiont was $F$. symbiotica, and reciprocally, the outgroup for F. symbiotica was R. insecticola.

\section{Phylogenetic reconciliations}

We used reconciliation analyses as implemented in Jane 3 [62] to infer cospeciation and host shift events along the evolutionary history of each symbiont. The history of symbiotic relationships is commonly disclosed by comparing host mitochondrial phylogeny and symbiotic phylogeny. Many studies use phylogenetic congruence between these two types of genomes to elucidate patterns of symbiotic inheritance [63, 64]. However, achieving a high resolution in reconstructing host phylogenetic information for closely related lineages from mitochondrial DNA is challenging [28]. Since the primary endosymbiont $B$. aphidicola is known to be strictly maternally inherited [65], our strategy to overcome this limitation was to use its phylogeny as a proxy for the host mitochondrial phylogeny. B. aphidicola is known to have a high-mutation rate [66] as highlighted in [32] and therefore appears to be a good indicator of the recent host history [63]. In reconciliation analyses, the parasite phylogeny (in our case, the secondary symbiont) is "mapped" onto the host phylogeny (i.e., each node in the parasite tree is assigned to a node in the host 
phylogeny). In such a map, the diversification events of the parasites are linked to their host phylogenetic history, so that four types of events are considered: cospeciation events, host switches, sorting events, and duplication events. For the host phylogeny, we used the matriline phylogeny inferred for $B$. aphidicola gene set data which showed a better resolution than the aphid mitochondrial phylogeny, and tested for each secondary symbiont whether primary and secondary symbiont phylogenies showed significant cospeciation (indicative of vertical transmission), using gene-based phylogeny for $S$. symbiotica, $H$. defensa, and $R$. insecticola and neighbor joining analysis of whole genome variants for $F$. symbiotica, Spiroplasma sp., Rickettsia sp., and R. viridis. For each cospeciation analysis, we first pruned aphid samples for which the focal symbiont was detected but had insufficient read coverage to obtain reliable data for phylogenetic inferences (i.e., we did not consider the symbionts in a sample when their coverage was comprised between $1 \times$ and $10 \times$ ), in order to avoid overestimating losses in the reconciliation process (i.e., considering that a symbiont was absent in an aphid sample while it was actually present but with insufficient data to perform a reliable variant calling). The focal symbiont was considered as absent when the coverage was inferior to $1 \times$. We ultrametrized the host and symbiont trees using Grafen's method using Ape package in R. We then ran Jane 3 [62] with the number of "generations" (iterations of the algorithm) set to 100 and the "population" (number of samples per generation) set to 100 and used default cost setting (cospeciation $=0$ and all other events $=1$ ). The cost of the best solution was compared to the distribution of the costs found in 500 randomizations in which the tip mappings were permuted at random. When the cost of the observed reconciliation is lower than expected by chance, the cospeciation signal is significant.

\section{Results \\ Most of the microbiome diversity is captured by the mapping approach}

On average, $90 \%$ of the reads were assigned by mapping to the pea aphid nuclear or mitochondrial genomes. The nuclear genome average coverage was $13 \times$ for individual sequencing and $66 \times$ for pool sequencing. $5.62 \%$ of the reads mapped on the genome of $B$. aphidicola and its plasmids, with an average coverage of $628 \times$ for individual sequencing and $3,694 \times$ for pooled sequencing. The coverages for the different secondary symbionts were very diverse and ranged from 0 (secondary symbiont was absent) to $1,300 \times$ (see Additional file 1). Presence and absence of symbionts as inferred from read depth was in agreement with the results of PCR diagnostic tests conducted for individual samples [42], and the few mismatches observed in the previous study were corrected by the choice of more appropriate reference sequences for Rickettsia sp., $R$. viridis, and Spiroplasma sp.

To further ensure that the used reference genomes were appropriate, we looked at the proportion of the genome covered by metagenomics reads and the average edit distance of reads mapping on each symbiont genome (minimum number of editing operations between the read and the corresponding part of the reference genome). Overall, more than $97 \%$ of the genomic positions of our reference genomes were covered by at least five reads. For F. symbiotica, we also checked that the mean edit distance of mapped reads was not larger than that of other symbionts for which we had reference genomes or we did a de novo assembly. Mean edit distance was 1.43 for F. symbiotica and ranged between 0.71 and 4.0 for other symbionts (average value was 1.67). Apparently, the use of a F. symbiotica genome assembled from another aphid host did not hamper the quality of the mapping.

Sequencing depth data are summarized in a presence/ absence matrix, as seen in Fig. 1 and are fully detailed in Additional file 1 . Since only a few infected individual aphids were enough to enable the detection of a symbiont in a pooled sample, pooled data generally contained a higher richness in secondary symbionts (on average 4.28 secondary symbionts per sample for pooled samples compared to 1 for individual sequencing).

\section{A low number of unmapped reads validates the mapping approach}

A few reads did not map onto any reference genome. The average rate of reads that did not map after quality control was $0.82 \%$ (median $0.62 \%$, min $0.25 \%$, $\max 4.76 \%$ ). It confirms that mapping metagenomic reads on this set of reference genomes is able to capture most of the genomic diversity of the pea aphid complex. The unmapped rate was heterogeneous between samples and appeared linked to the symbiotic composition of the samples. Samples infected by symbionts for which a draft reference genome was used for mapping (Spiroplasma and Rickettsia) contained more unmapped reads. These reads probably originate from genomic regions absent or too divergent from these draft reference genomes. When considering samples containing only symbionts with good quality and closely related genomes, the average unmapped rate lowered to $0.69 \%$.

The nature of those unmapped reads was further explored by conducting a taxonomic assignation of such reads with Centrifuge (version 1.0.3) [51] and its default database. Overall, only $4.9 \%$ of the unmapped reads were assigned to a taxon. The taxonomic assignation of unmapped reads is summarized in 


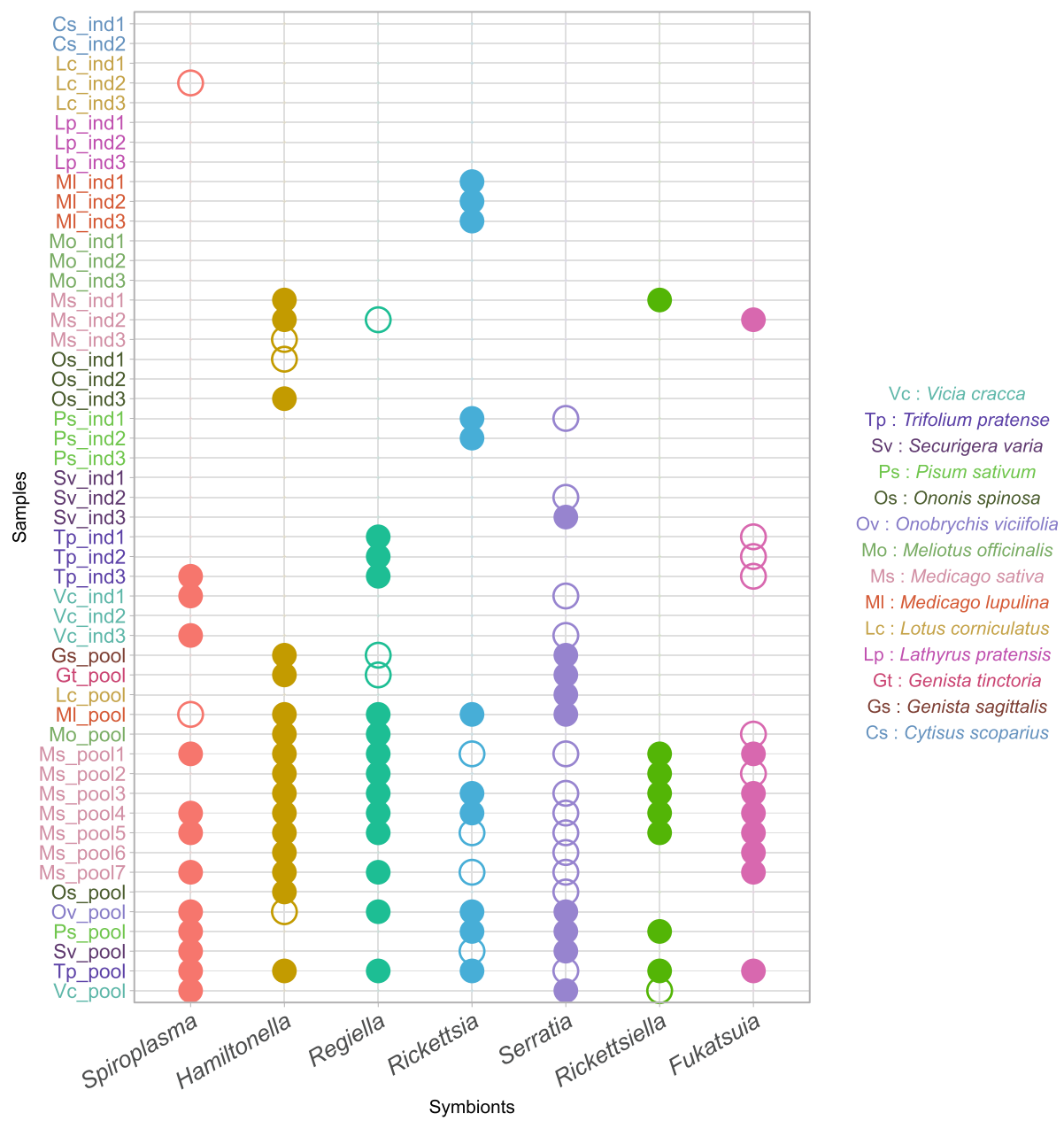

Fig. 1 Presence/absence pattern for bacterial symbionts as detected in the metagenomic dataset. Pea aphid individuals (ind) and populations (pool) were analyzed. Empty circles indicate a coverage greater than $1 \times$. Filled circles indicate a coverage greater than $10 \times$, enabling phylogenetic analysis. A. pisum and Buchnera aphidicola genomes were detected in every sample

Additional file 4 and can be explored for all samples on the website https://aphid-microbiome.netlify.com/. It is in accordance with mapping results. Some reads of host or symbiotic origins that were not mapped to the appropriate reference genome were however accurately assigned by Centrifuge. Other taxa were also found by Centrifuge assignation, either because of over-assignation by the program or because some environmental organisms were sequenced along with the pea aphid and its symbionts. These reads represented a small fraction of the unmapped reads. Most unmapped reads were not taxonomically assigned by Centrifuge, probably because they contained sequencing errors or were too distant to any reference sequence in the Centrifuge database. Overall, these results indicate that the microbiota of the pea aphid complex is dominated by a few heritable symbionts and that we achieved a close to exhaustive inventory of the microbiome of our pea aphid samples.

\section{Different levels of intra-specific diversity for the pea aphid symbionts}

The overall genomic diversity of the selected samples was estimated for each symbiont by measuring the density of variable sites between the two most different symbiont genomes in the dataset. Only pooled samples were considered in this analysis, in order to have a more comparable sample size for each symbiont.

Variant calling results are summarized in Table 2. They show strong contrasts in genomic diversity between the different symbiont taxa associated with the pea aphid complex. H. defensa and R. insecticola showed the highest diversity, with 12.6 and 16.8 variants per kilobase (kb), respectively. Conversely, genomic diversity was extremely low for $R$. viridis, with an average of 0.027 variants per kb. The other symbionts (B. aphidicola, F. symbiotica, Spiroplasma sp., Rickettsia sp., and S. symbiotica) showed intermediate levels of genomic diversity (with respectively 3.0, $1.59,1.28,1.19$ and 1.0 variants per kb). Consequently, the 
Table 2 Summary of variant calling results. Outgroup samples were excluded to report the diversity within the dataset

\begin{tabular}{lllll}
\hline Symbiont & Number of samples & Number of SNPs/kb & Number of indels/kb & Maximum distance between two samples (variants/kb) \\
\hline Serratia symbiotica & 9 & 1.46 & 0.13 & 1.00 \\
Buchnera aphidicola & 50 & 12.61 & 0.56 & 3.03 \\
Hamiltonella defensa & 16 & 22.16 & 0.91 & 12.61 \\
Regiella insecticola & 12 & 18.20 & 0.56 & 16.75 \\
Rickettsia sp. & 9 & 1.19 & 0.12 & 1.19 \\
Rickettsiella viridis & 8 & 0.03 & 0.00 & 0.03 \\
Fukatsuia symbiotica & 8 & 2.21 & 0.04 & 1.60 \\
Spiroplasma sp. & 12 & 1.95 & 0.00 & 1.28 \\
\hline
\end{tabular}

lengths of the branches of the phylogenetic trees built for these various symbionts were highly variable.

\section{Phylogenomic analysis of Buchnera aphidicola from the pea aphid complex}

By analyzing genomic variation over the whole genome of $B$. aphidicola, we built a well-supported phylogeny of the pea aphid obligatory symbiont. No substitution saturation was detected using the Xia's test [57] (see Additional file 5). Figure 2 shows the results of the phylogenomic analysis for $B$. aphidicola across all datasets, using maximum likelihood-based inference on a 96 gene set alignment. The tree topology obtained from the gene set was compared with a whole genome variant profile clustering. Overall, the two phylogenetic methods gave similar results, as shown in Additional file 6. The few mismatches observed between the two topologies mainly involved nodes with low support in both trees.

As previously observed using partial sequences of pseudogenes data [33], B. aphidicola genomes associated with the pea aphid complex are separated into two distinct clades.

Matrilines from the same biotype were generally clustered together, but some were scattered across the phylogeny (e.g., Vicia cracca and Ononis spinosa biotypes did not form single clusters). The fact that some samples from the same biotype did not cluster together likely results from incomplete lineage sorting or ongoing gene flow between biotypes [32]. When comparing $B$. aphidicola and mitochondrial phylogenies (see Additional file 7), the well-supported branches of the latter were identically retrieved on the endosymbiont phylogeny, but $B$. aphidicola phylogeny was better resolved. This confirms the suitability of using $B$. aphidicola phylogeny as a framework for examining evolutionary dynamics of secondary symbiont infections. Overall, we built a solid phylogenetic framework for $B$. aphidicola with good branch supports that we further used to contrast primary and secondary symbiont histories.

\section{Phylogenetic insights on the evolutionary histories of host-secondary symbiont associations}

We then examined the evolutionary histories of the associations between secondary symbionts and their pea aphid hosts by comparing one by one the matriline phylogeny reconstructed from $B$. aphidicola with the phylogeny of each of the seven secondary symbionts detected with sufficient coverage in our metagenomics dataset.

Visual comparison of the matriline phylogeny with $H$. defensa phylogeny revealed some congruent nodes but also several differences in tree topologies indicating frequent horizontal transfers of this symbiont in the pea aphid complex (Fig. 3). Reconciliation analyses detected nine possible events of host shifts and six cospeciation events, which yielded a co-diversification scenario that is less costly than expected by chance. In addition, three events of loss were detected. This reflects mixed patterns of transmission with overall vertical transmission of this secondary symbiont along the evolutionary history of the pea aphid complex, combined with multiple events of horizontal transfers and some losses (see Additional file 8). Spiroplasma sp. phylogeny also showed many incongruencies with the matriline phylogeny, presumably reflecting frequent horizontal transfers (Fig. 4). Reconciliation analysis inferred eight potential host-switch events and only three cospeciation events (see Additional file 8). In that case, the cospeciation hypothesis was rejected, indicative of a shorter association of Spiroplasma with the pea aphid complex.

The $R$. insecticola phylogeny retrieved two well-differentiated clades (Fig. 5). Whole genome variant calling indicated that more than 30,000 variants distinguish these two clades, while intra-clade variation was much lower, with at best 8000 variants called. These two clades may have infected the pea aphid complex separately and seem to be preferentially associated with different biotypes (Medicago sativa for clade 1 and Trifolium for clade 2). Given the low variation within each lineage relative to the large divergence between the two lineages, we can confidently assume that the acquisition of these symbionts by the different aphid hosts occurred after their divergence. The matriline phylogeny and the $R$. insecticola phylogeny showed several incongruencies 


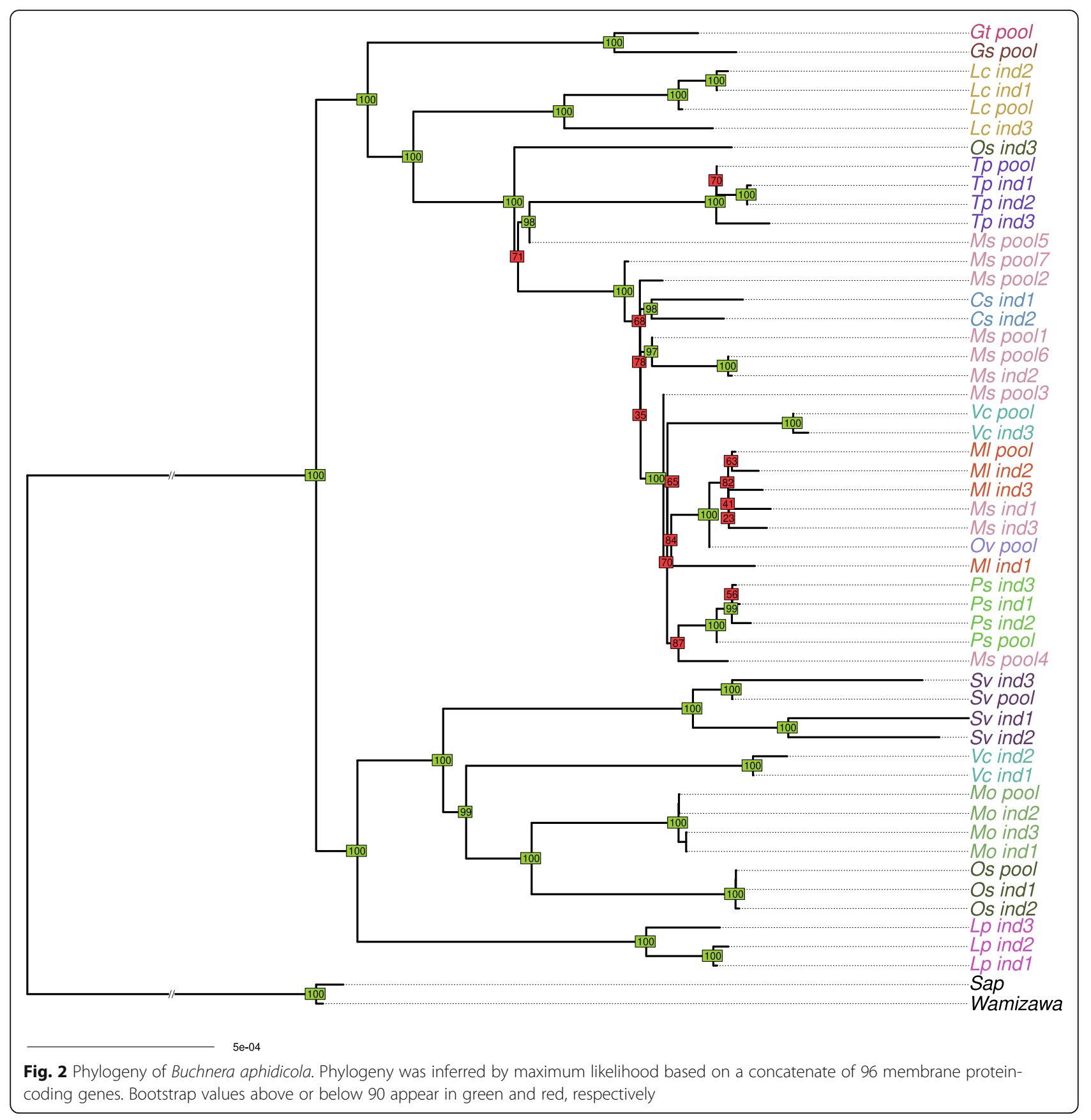

within and between the two clades, suggesting frequent horizontal transfers, as suggested above for $H$. defensa and Spiroplasma. Accordingly, the reconciliation analysis detected 10 events of host switch and a single cospeciation event. The signal of cospeciation between Regiella and Buchnera was not significant, supporting horizontal transmission and frequent losses of events of this symbiont in the pea aphid complex (see Additional file 8).

Despite of the low genomic diversity found for $R$. viridis, most nodes of the phylogeny are well supported (Fig. 6). Reconciliation analysis revealed only one cospeciation event along with six host-switch events. Accordingly, no significant cospeciation signal was found. This result, combined with the fact that this symbiont was found in only three biotypes of our sample and is poorly diverse, suggests a very recent history of this association in the pea aphid complex. In our sample, F. symbiotica was associated preferentially with the Medicago sativa biotype, either because of its recent acquisition, low rate of horizontal transfers, or strong incompatibilities/counter-selection in other biotypes. Phylogenetic analysis revealed a few incongruencies between tree topologies of host matrilines and 


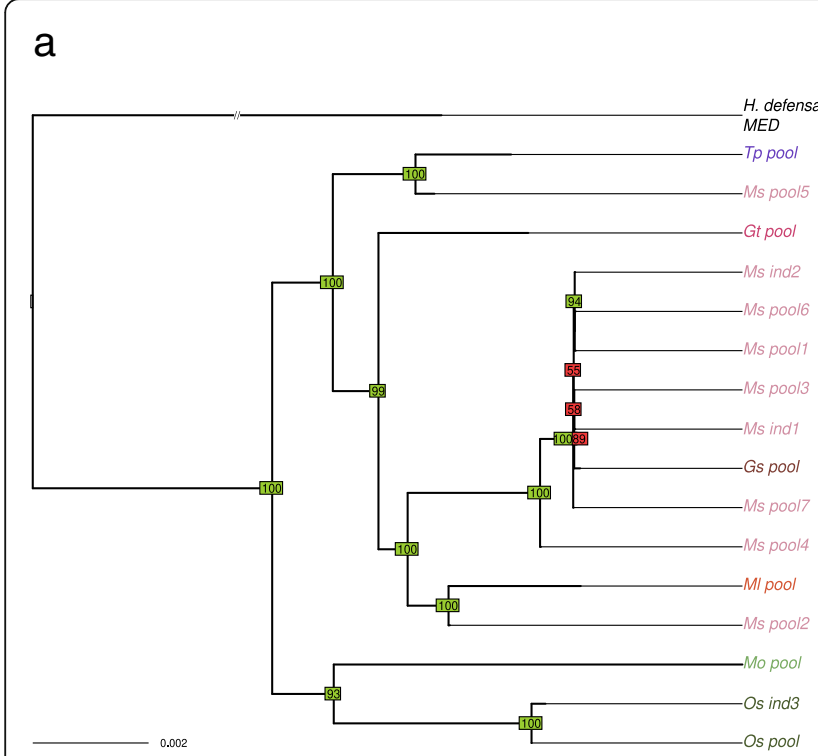

b
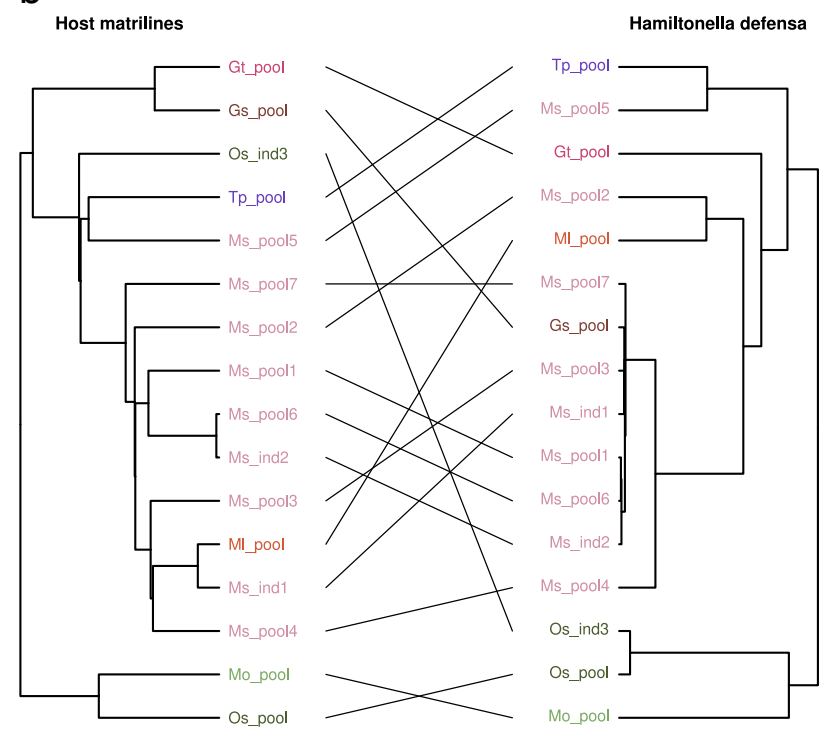

Fig. 3 a Phylogeny of Hamiltonella defensa. Phylogeny was inferred by maximum likelihood based on a concatenate of 118 membrane proteincoding genes. Bootstrap values above or below 90 appear in green and red, respectively. b Tanglegram of Hamiltonella defensa phylogeny with host matriline phylogeny

F. symbiotica (Fig. 7). This pattern presumably reflects cases of horizontal transfer, in agreement with the reconciliation analysis that detected three host switch events. However, we found a significant signal of cospeciation (four putative events), indicative of overall vertical transmission within the Medicago sativa biotype.

Several incongruencies were observed between the phylogenies of Rickettsia sp. and B. aphidicola (Fig. 8).
The reconciliation analyses uncovered four host switch events and four cospeciation events; the cospeciation signal was not significant.

The S. symbiotica phylogeny delineated several clades for this symbiont (Fig. 9). Nine samples were infected by this symbiont in eight different biotypes, indicating that S. symbiotica is represented in most of the biotypes but at a moderate prevalence across the complex. Some incongruencies were observed between the S. symbiotica and primary

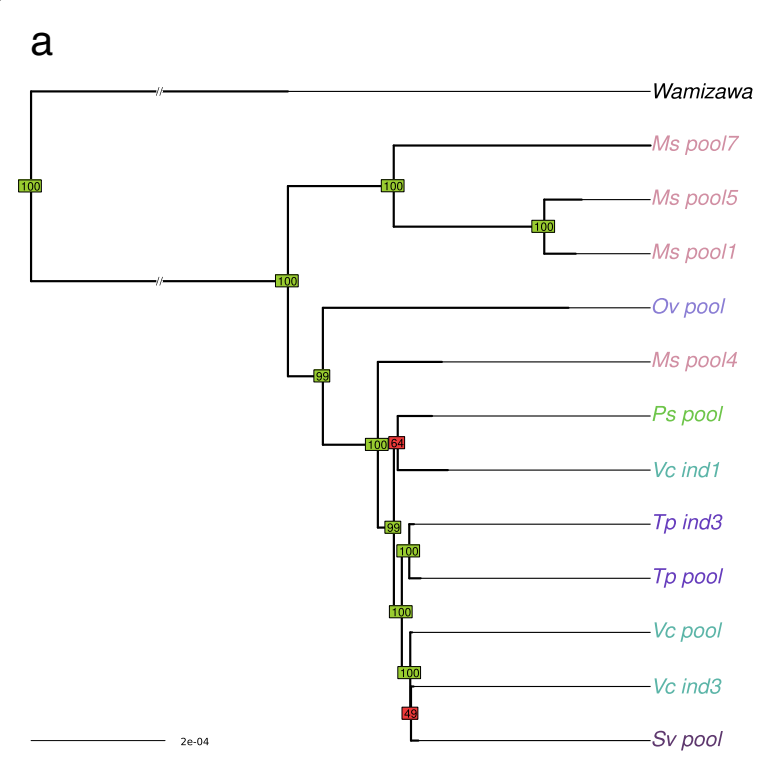

b

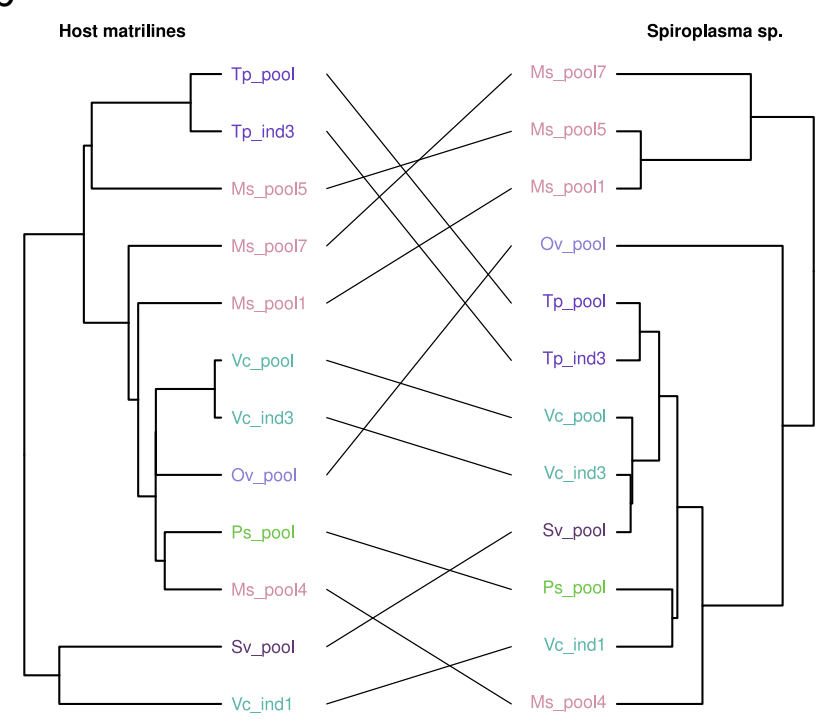

Fig. 4 a Phylogeny of Spiroplasma sp. Phylogeny was inferred by neighbor joining based on a whole genome variant calling. Bootstrap values above or below 90 appear in green and red, respectively. b Tanglegram of Spiroplasma sp. phylogeny with host matriline phylogeny 
a

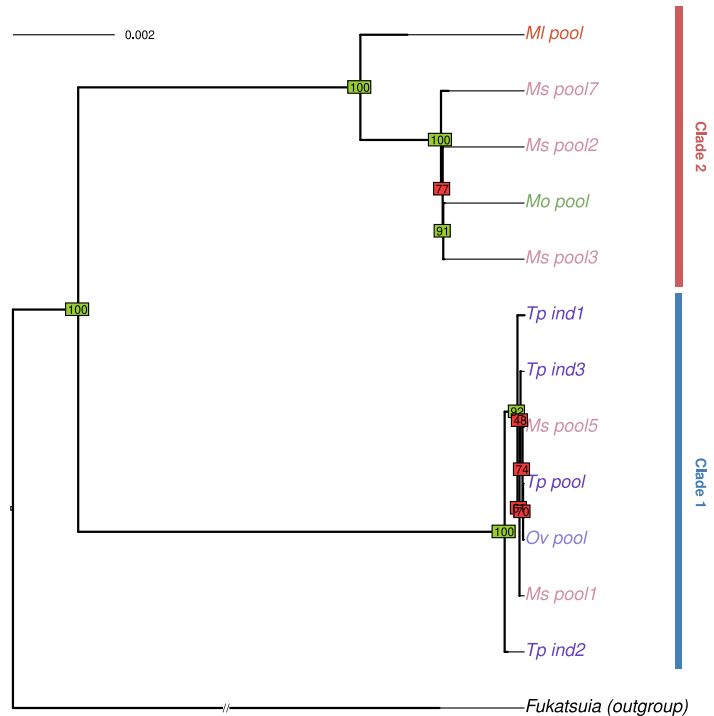

b

Host matrilines

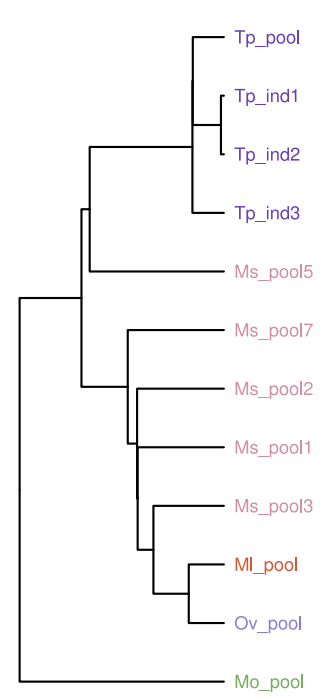

Regiella insecticola
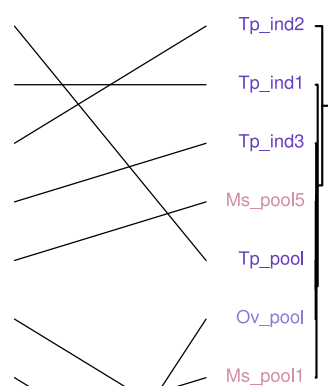

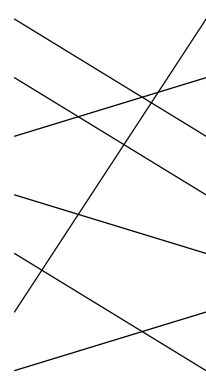

Ms_pool7

Ml_pool

Fig. 5 a Phylogeny of Regiella insecticola. Phylogeny was inferred by maximum likelihood based on a concatenate of 96 membrane proteincoding genes. Bootstrap values above or below 90 appear in green and red, respectively. b Tanglegram of Regiella insecticola phylogeny with host matriline phylogeny

symbiont phylogenies but all involved nodes with low support on the S. symbiotica phylogeny. Reconciliation analyses revealed a few number of host switch events along with significant cospeciation (Additional file 8). However, the fact that S. symbiotica is found at a moderate prevalence suggests some failures in vertical transmission, leading to loss events in pea aphid lineages (three losses were indeed detected by the reconciliation test).

Intra-host coexistence of two Regiella insecticola strains Investigation of inter-sample phylogenetic relationships was led by considering the most abundant alleles for each sample. However, some samples may be polymorphic at

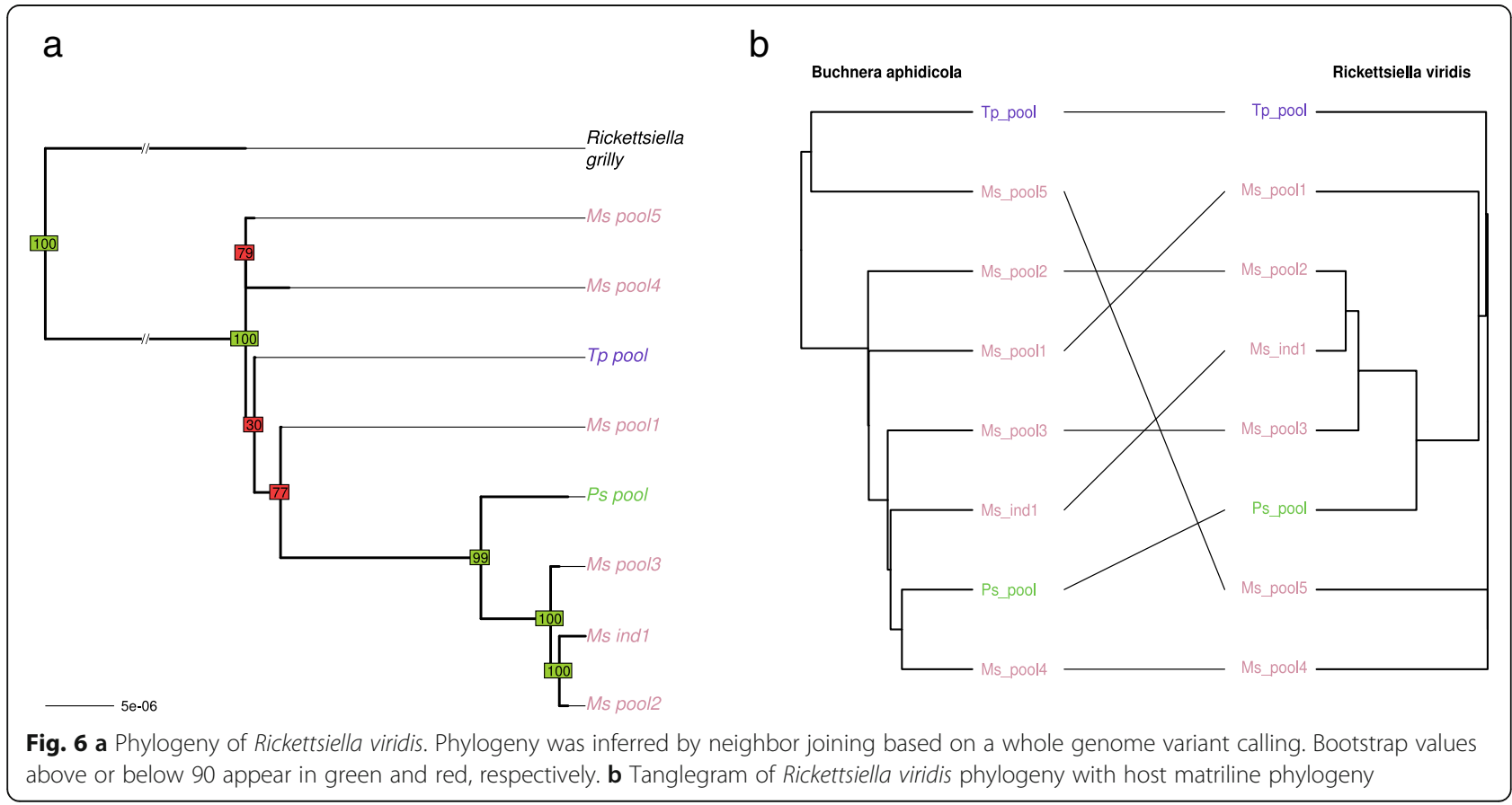




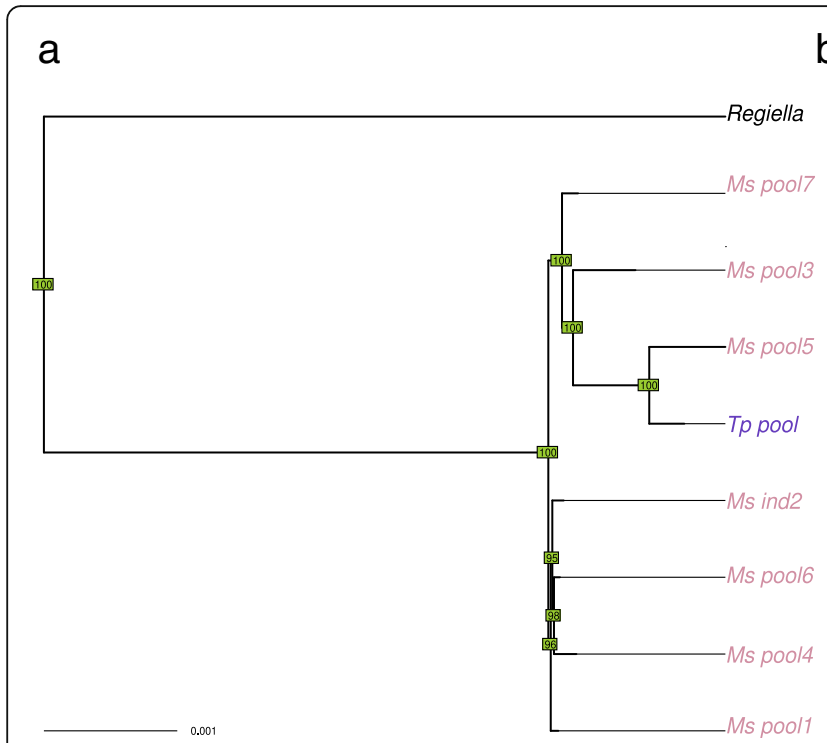

b

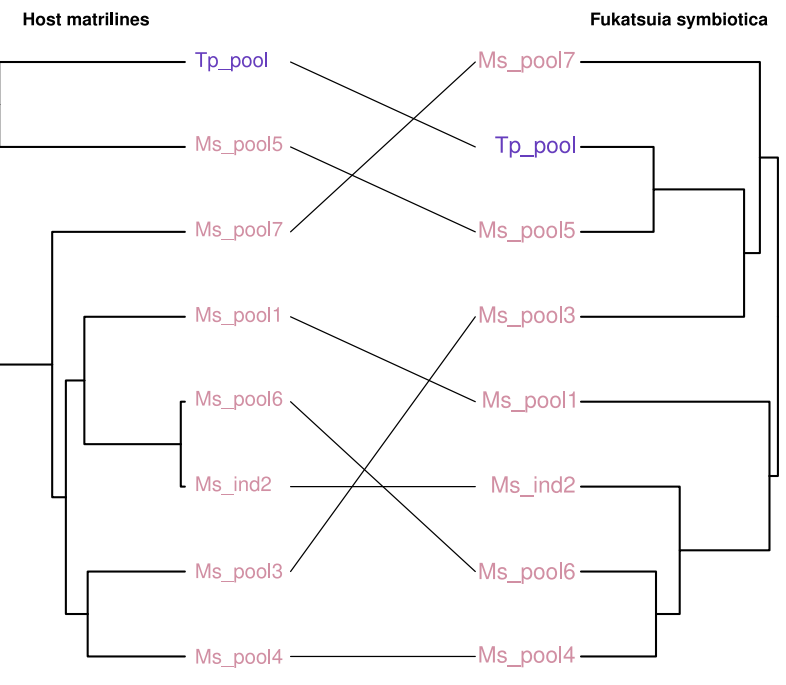

Fig. 7 a Phylogeny of Fukatsuia symbiotica. Phylogeny was inferred by neighbor joining based on a whole genome variant calling. Bootstrap values above or below 90 appear in green and red, respectively. b Tanglegram of Fukatsuia symbiotica phylogeny with host matriline phylogeny

some sites, with both the reference and alternative alleles covered in metagenomic dataset. While intra-sample genomic variability is expected for pooled samples, which originated each from a diverse host population, it would be more surprising for individual sequencing samples. However, we observed that genome sequences from two distinct clones of the Trifolium biotype (Tp_ind1 and Tp_ind2) showed a high number $(32,000)$ of intra-sample polymorphic sites along the $R$. insecticola genome. These two samples showed no sign of polymorphism for the primary symbiont and mitochondrial genomes, excluding the hypothesis of contamination during the sequence data production.
Figure 10 shows the coverage distribution for major and minor alleles of $R$. insecticola in the Tp_ind1 sample. A similar distribution was obtained for the Tp_ind2 sample. These bimodal distributions suggest that two genotypes of $R$. insecticola coexist in these two samples. We estimated the read depth of the two genotypes with the most abundant genotype in Tp_ind1 covered at around $40 \times$ and the other genotype at around $10 \times$ (respectively $25 \times$ and $10 \times$ for Tp_ind2). The variant profiles for these two genotypes were close to the ones observed for the two clades of $R$. insecticola described in Fig. 5.

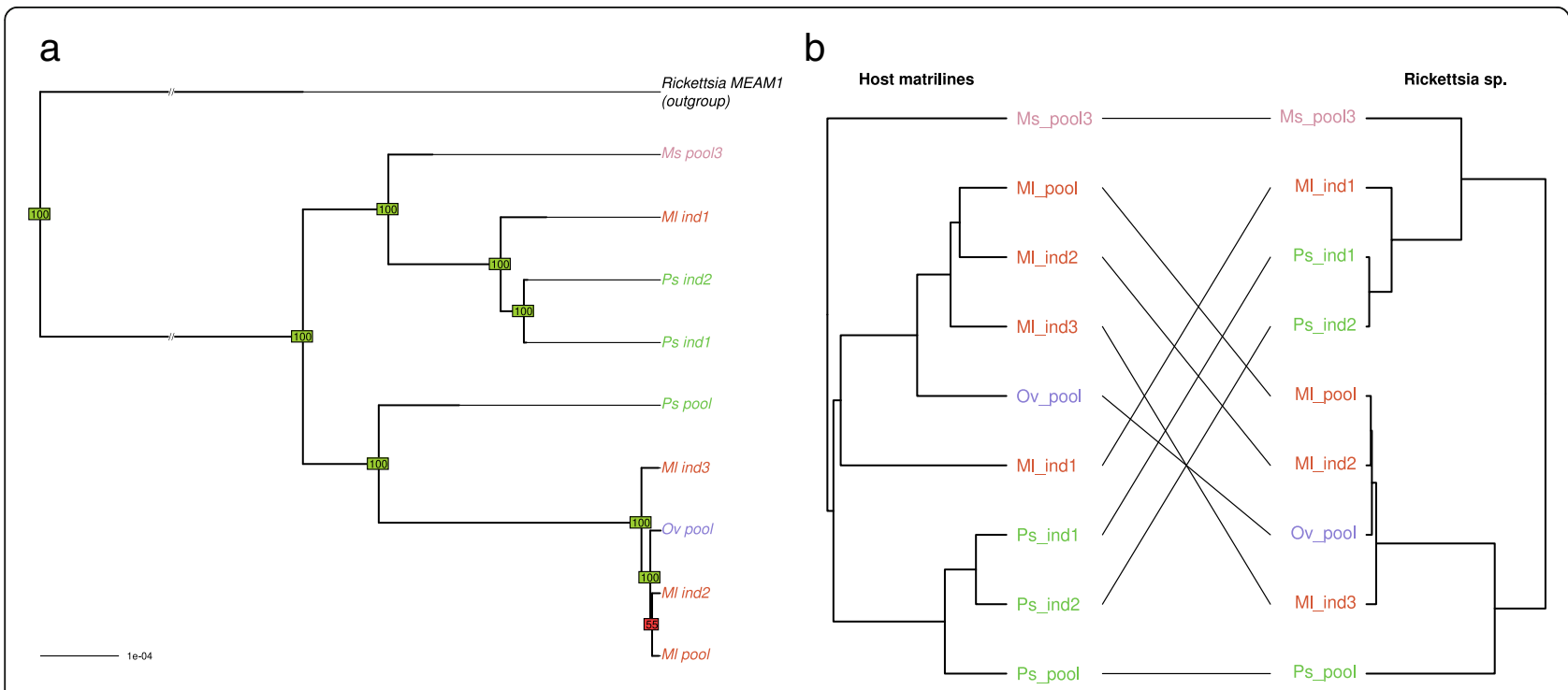

Fig. 8 a Phylogeny of Rickettsia sp. Phylogeny was inferred by neighbor joining based on a whole genome variant calling. Bootstrap values above or below 90 appear in green and red, respectively. b Tanglegram of Rickettsia sp. phylogeny with host matriline phylogeny 


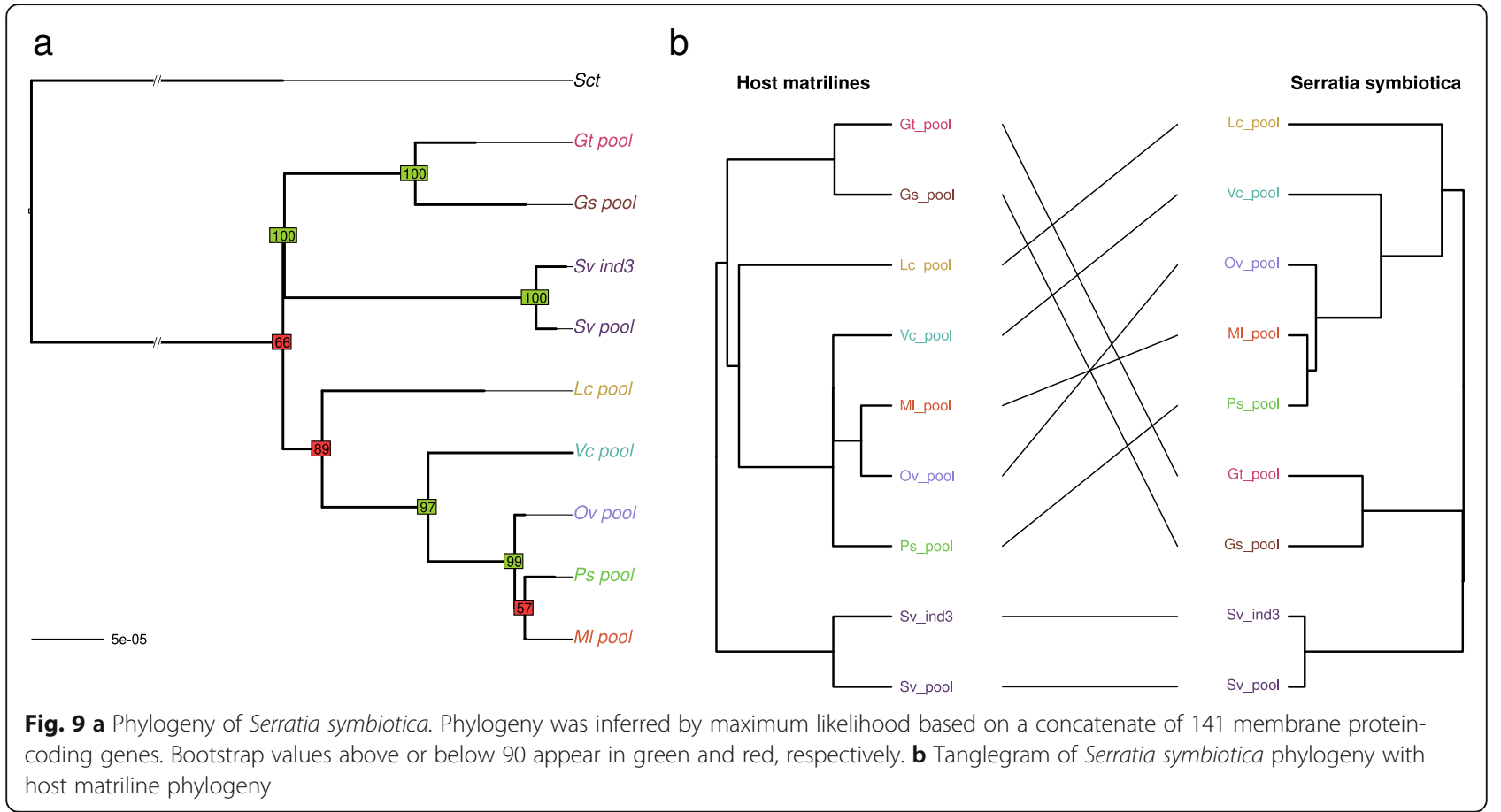

Sequencing data thus indicate the coexistence of two $R$. insecticola lineages inside particular samples, but it does not prove this coexistence inside individual aphids, because samples denominated as "individual sequencing" actually resulted from the sequencing of a pool of individual aphids from the same clone. Therefore, it is possible that aphids from the same clone host different symbiont genotypes. To challenge this hypothesis, we

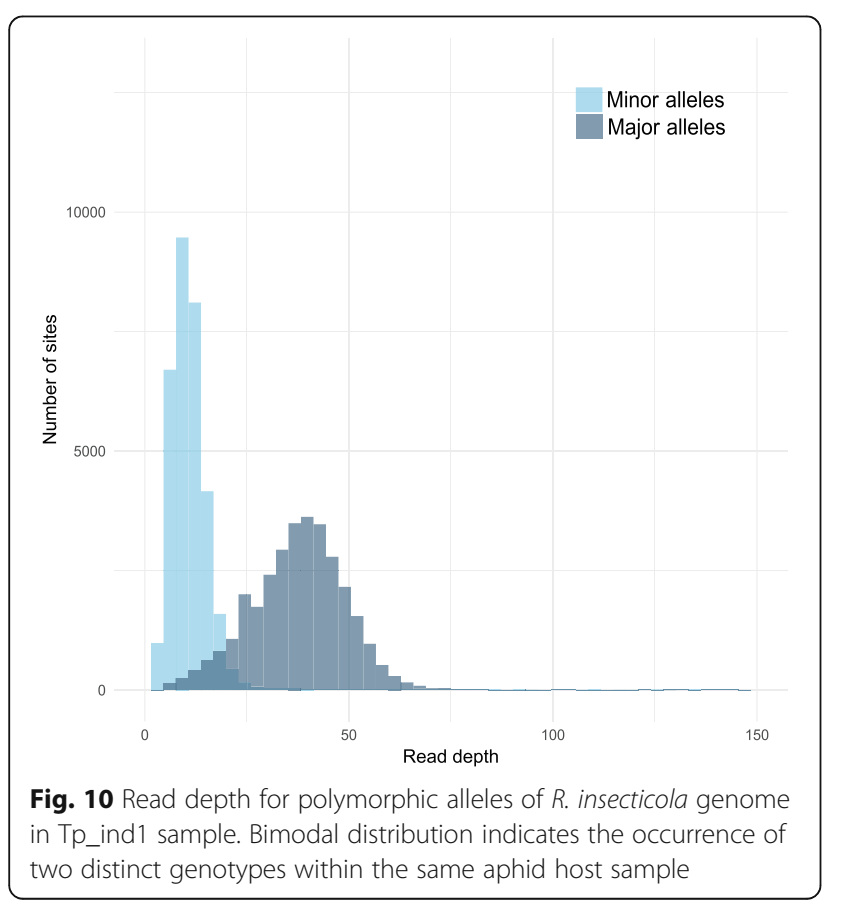

performed experimental validation on individual aphids picked in the clonal lineage maintained in culture in our laboratory. A deletion of $32 \mathrm{bp}$ differentiating the two clades was identified on the contig of accession AGCA01000518 (see Additional file 9). We designed primers to amplify the region corresponding to this deletion. Electrophoresis confirmed the presence of the two haplotypes in individual aphids from the Tp_ind1 and Tp_ind2 clonal lineages, while a single haplotype was detected in aphids from the Tp_ind3 clone (Additional file 9: Figure S9). This validation confirmed the coexistence inside single individual aphid hosts of two distinct genotypes of $R$. insecticola.

\section{Discussion}

We present here a framework to explore multi-scale genomic diversity in holobiont systems of low complexity, which is generally the case of insect holobionts. We applied this approach to metagenomic datasets of the pea aphid complex by considering microbial variation across host biotypes, among individuals of the same biotype and within individual aphids. This work allowed to extract more than $99 \%$ of the metagenomic information and to draw a complete inventory of microbes associated to the pea aphid complex, revealing a microbiota dominated by a few bacterial symbionts. Our approach also revealed for the first time a large genomic diversity among A. pisum symbionts, with different diversity patterns between symbiont taxa presumably reflecting distinct evolutionary histories, genomic features, transmission patterns, and ecological influences across pea 
aphid biotype-symbiont associations. Finally, phylogenomic analyses highlighted that frequent horizontal transfers and losses of facultative symbionts have probably been common events during the diversification of the A. pisum complex.

\section{Guidelines for analyzing multi-scale holobiont metagenomic diversity}

The method proposed to finely analyze holobiont metagenomic diversity was based on the mapping of metagenomic reads on a set of reference genomes. By doing so, the entangled metagenomic read set was transformed into symbiont-specific read subsets, which enabled finer analyses such as intra-sample variability detection or strain-level diversity analyses. The method is reliable for the pea aphid holobiont, which has a restricted number of symbiotic partners, and for which reference genomes are partly available. The rate of unmapped reads was below $1 \%$ for most samples, and variations depending on the composition of symbiotic communities were observed, indicating that the availability and quality of reference genomes are important to achieve a good assignation of the metagenomic reads. When distant reference genomes are used for mapping, highly divergent regions and large insertions or deletions obviously limit the assignation success rate. Overall, mapping of metagenomics reads on a set of reference genomes (when available) or de novo assembled genomes (when coverage is sufficient), followed by a strain-level analysis of genomic variation appears to be an appropriate characterization method in the case of the pea aphid holobiont.

A large number of aphid samples were sequenced in order to investigate microbial diversity across the pea aphid complex of biotypes. However, sequencing data from host aphids did not allow accessing directly to individual bacterial genotypes, and we had to build genotypes based on the most abundant alleles in each bacterial population. In our dataset, individual sequencing samples had either a low intra-sample polymorphism or a mix of genotypes we could easily disentangle (as for example $R$. insecticola in the Trifolium biotype). However, pooled samples were analyzed so that only the most abundant alleles were kept to reconstruct the genotype of each symbiotic lineage. Overall, this assumption leads to underestimate the actual diversity in the pooled samples. Compared to individual host genotype sequencing, pooled sequencing allows to capture a greater diversity of symbiotic lineages, but suffers limitations in reconstructing individual bacterial genotypes, due to methodological problems in handling large intra-sample polymorphism. Despite this limitation and the fact that we applied stringent filters to discard ambiguous variants, in most cases, we could retrieve a sufficient number of reliable variants from metagenomics reads to compare symbiont diversity and to build well-resolved phylogenies.

Search for genomic variants was restricted to SNPs and short insertions and deletions. The analysis of large genomic rearrangements may bring additional information on the symbiotic genomic diversity [67]. Short variant information seems to be sufficient to reconstruct symbiotic phylogenetic trees, since most phylogenetic studies rely on gene sequences analyses, and generally do not integrate rearrangements, but this structural variation should not be neglected in order to reconstruct full genomes for the main microbial genotypes existing in pea aphid holobionts.

\section{Multi-scale diversity inventory of an holobiont}

Previous studies on the pea aphid's microbiota focused on the detection of symbionts using 16S rRNA PCR-based detection or $16 \mathrm{~S}$ amplicon sequencing [34, 39]. The drawbacks of these methods are that they are restricted to bacteria, have generally low taxonomic resolution, suffer from several biases due to DNA amplification, and may be unable to identify new microbial partners [68].

To overcome these limitations, we used shotgun metagenomic sequencing, which captures whole genomic information about the host and its associated microbial community. We successfully assigned most of the reads to host and symbiont reference genomes forming the pea aphid holobiont and checked that no new bacterial symbiont was abundant in unmapped reads. Also, we found no evidence for the occurrence of Wolbachia in our large metagenomic dataset though this symbiont has been reported in A. pisum in three previous studies [34, $69,70]$. One explanation could be that none of the pea aphids used for individual or pooled resequencing projects was infected by this symbiont. Alternatively, detection of Wolbachia in previous studies could result from artifacts or DNA amplification from aphid endoparasitoids which may be infected by this symbiont. Because DNA was extracted from aphid clonal lineages cultured in laboratory conditions for two generations (to avoid contamination from aphid parasite microbiota and limit environmental microbes), only the inherited part of the microbiota was sequenced. In contrast with a previous study based on $16 \mathrm{~S}$ rRNA sequencing [34], no gut associate microbe was found in our metagenomic dataset, suggesting either a low prevalence of such microbes in pea aphid populations, their loss in culture because of poor vertical transmission, or an artifact of $16 \mathrm{~S}$ rRNA data. Finally, apart from the bacteriophage APSE, no fungal or viral associates were found. However, because of their small genome sizes, unreferenced viruses could have been missed in the unmapped-reads analysis. In 
addition, RNA viruses are common in arthropods and need specific detection methods [71]. Therefore, further analyses are required to in depth examination of the pea aphid virome with dedicated approaches [71]. These results altogether indicate an apparent low complexity of the pea aphid microbiota when considered at a species-level scale and are in accordance with previous works on aphids and other sap-feeder insects showing low richness of host-associated microbial communities and mostly composed of a few heritable bacterial symbionts [72, 73].

\section{Contrasting evolutionary dynamics of pea aphid- secondary symbiont associations}

The history of the symbiosis between aphids and their primary symbiont $B$. aphidicola is well known, with a 160-280 million years old association [74]. Although $B$. aphidicola can be experimentally transferred between aphid matrilines and has been lost in a few aphid taxa [75], it is considered as a strictly maternally inherited symbiont, and no horizontal transfer has been observed so far at different phylogenetic scales [63, 76, 77]. For $A$. pisum, we observed in the present work a close congruence between mitochondrial and $B$. aphidicola phylogenies, indicating a persisting association between the host and its primary symbionts, and a codiversification of both partners in recent evolutionary time. Genome-wide analysis of $B$. aphidicola diversity in the pea aphid complex showed a diversification of pea aphid matrilines which corresponds well to the adaptive radiation that led to the complex of biotypes and confirmed previous results obtained from pseudo-gene sequences of Buchnera [33, 66]. Using our well-resolved $B$. aphidicola phylogeny, we were able to contrast the evolutionary trajectories of pea aphid matrilines with that of every $A$. pisum secondary symbiont and to propose different history scenarios of pea aphid-secondary symbiont associations.

Several secondary symbionts are known in A. pisum and other aphid species, but the nature of their association with aphid hosts is variable, from free association to co-obligatory symbiont with intermediate stages of dependency [44]. Recent data provide evidence for a higher rate of mother to offspring transmission for most of the secondary symbionts presented here [78], but some indirect proofs of horizontal transfers have also been reported [29, 35]. Their underlying mechanisms are still unclear, with host plant, natural enemies, or paternal transmission as candidate paths for horizontal transfers [30]. In this study, we showed a contrasting genomic diversity for the different symbionts, from poorly diverse symbionts such as Rickettsiella viridis to highly heterogeneous ones such as Regiella insecticola. This heterogeneity in genomic diversity could result from the combination of several factors, such as differences in evolutionary rates, population size, transmission modes, and host-symbiont association histories [78-80]. It is also very likely that these symbiotic associations are constrained by different factors including host compatibility to new infection [81] and selection [35]. For example, some symbionts like Serratia seem to have a wide host range [82] while others like Fukatsuia tend to be more restricted in terms of biotypes. In the specific case of $R$. viridis, although we cannot totally discard this hypothesis, the very low genomic variation is unlikely to result from a low-mutation rate considering the level of diversity of $R$. viridis which is two orders of magnitude less than for the other symbionts associated to pea aphids and that there is no particular mention of this pattern in the literature. Instead, this low-population genomic diversity in $R$. viridis might rather result from its relatively recent acquisition by a few $A$. pisum lineages, likely from a single of a small number of sources.

Evolutionary dynamics of symbiotic associations in the pea aphid complex were studied here by comparing phylogenetic trees of secondary symbionts with that of the obligatory symbiont $B$. aphidicola, as a proxy of pea aphid matriline phylogeny. While symbiotic species showing phylogenetic congruence with $B$. aphidicola probably reflect co-speciation with their aphid host lineages, incongruent symbiont phylogenies are expected to result from different events such as horizontal transfers or symbiont loss/gain events. Accordingly, incongruencies between matriline and secondary symbiont phylogenies were observed for all secondary symbionts considered in this study. Host switches were detected for every secondary symbiont by reconciliation analyses, supporting the hypothesis of frequent horizontal transfers proposed in previous studies on that system [35]. Reconciliation analyses also detected a few events of loss for most symbionts and those could result from failures in vertical transmission as sometimes observed in laboratory conditions [30]. With reconciliation analyses, we also found several cases of significant signals of co-speciation between secondary symbionts and their host matrilines. Since secondary symbionts of the pea aphid are maternally inherited with a generally good fidelity [78], this is not a surprising result. However, these results need to be interpreted with care as for some samples (pooling several individuals); we only reconstructed the most abundant genotype for each symbiont and might have therefore underestimated the phylogenetic diversity of the biotype-symbiont associations and the complexity of co-diversification scenarios. In any case, our approach suggests that cospeciation signals as well as the numbers of gain and loss estimated from reconciliation tests greatly differ between secondary symbionts, reflecting mixed patterns of transmission and 
different dynamics and durations of these symbiotic associations among the pea aphid complex. In the case of Regiella insecticola, we revealed an even more complex situation: $R$. insecticola populations in pea aphid biotypes encompass two highly differentiated genotypes, likely representing two distinct events of infection by symbiont strains that diverged much before the diversification of pea aphid biotypes. Horizontal transfers of these two genotypes were also detected within the pea aphid complex, indicating more recent host switch.

Overall, these evolutionary scenarios of symbiotic associations in the pea aphid complex suggest that the rate and source of horizontal transfers are very variable across symbionts, in accordance with previous studies at lower resolutions [29]. Yet, these results may be extended by larger phylogenetic studies in the pea aphid complex but also in other aphid and arthropod taxa, and by investigations of the amount and mechanisms of gain (horizontal transfers) and loss of secondary symbionts in natural populations of pea aphids.

\section{Intra-host coexistence of different Regiella insecticola strains}

Our metagenomic approach on the pea aphid microbiota also revealed an unexpected level of diversity. Indeed, this study showed evidence for the coexistence of two divergent $R$. insecticola genotypes within the same individual aphid. While the within-host coexistence of symbiotic strains from the same lineage has already been reported in some arthropods [83], it has been rarely found in aphids (but see [44]). This bi-infection of $R$. insecticola strains inside individual aphids has been observed for two clones, where the two existing strains were both very different and equally abundant, facilitating detection and characterization of their infection status. However, some less obvious cases of multi-infection in other samples or by other symbionts might have been undetected. The development of dedicated techniques to analyze intra-sample polymorphism may help to better understand these events of coinfection and their evolutionary implications. The discovery of this symbiotic coinfection raises new questions concerning the effects of these strains, individually or in conjunction, on host fitness and phenotype, their localization and interaction in the aphid host, and the stability of this coinfection.

An important aspect which requires dedicated studies is how this genomic diversity in pea aphid microbiota translates into functional differences and influences the holobiont phenotype. It is known that strain-level genomic variation can have considerable consequences on the expression of the host extended phenotype. For instance, previous works demonstrated that the level of natural enemy protection provided by $H$. defensa is highly different between two Genista biotypes infected by genetically distinct strains of the protective symbiont [37]. Here, the reconstructed $H$. defensa phylogeny confirmed that these two Genista biotypes host highly different symbiotic populations, while sharing close matriline history. Genome-wide variant discovery may help to infer metabolic differences between $H$. defensa genotypes and their associated APSE phages that could cause the variation in protection levels of the hosts [84]. Similarly, a functional annotation of the genomic differences between the two highly divergent genotypes of $R$. insecticola found singly or in co-infection within the same host, may reveal different impacts on the host phenotype.

\section{Conclusions}

We conducted a multi-scale analysis of genomic diversity associated with the pea aphid microbiota, ranging from the common species- and biotype-levels analysis, to a more innovative intra-specific analysis, and we were able to uncover the genomic diversity at each considered scale.

Improved understanding of host-microbiota relationships may benefit from large holobiont sequencing projects, and we believe the framework we developed here is applicable to other holobiont systems of low complexity. By analyzing whole genome variation in the pea aphid holobiont, we confirmed that its microbiome diversity is limited to a few inherited symbionts, but we revealed a generally large genomic diversity observed at different levels of the holobiont organization. This genomic diversity in populations of secondary symbionts seems to be mainly shaped by the dynamics of symbiotic associations, which could take multiple routes and lead to different evolutionary trajectories.

This work paves the way for new studies relying on metabolic and functional approaches and aiming to examine how genomic variation in microbiota affects host fitness and phenotypic traits. Moreover, a full understanding of the evolutionary history and ecology of symbiotic associations requires a larger investigation of the sources of genomic diversity at different geographical, temporal, and trophic scales.

Although the metagenomic framework we developed here for the pea aphid system yielded significant knowledge improvements in patterns of genomic diversity and evolution in host-symbiont associations, we pinpointed some limitations in our approach such as the availability of reference genomes and the difficulty to handle metagenomic data of high complexity. Methods to analyze fine-scale diversity from metagenomic dataset are still rare and require either well annotated reference genomes or simple communities where organisms are easy to disentangle. More advanced methods have to be 
developed to assess metagenomic diversity in either complex or non-model holobionts.

\section{Additional files}

Additional file 1: Read depth of reference genomes for each sample.
(XLSX $24 \mathrm{~kb})$

Additional file 2: Statistics of the symbiont reference genomes used for mapping and phylogenetic analyses. (DOCX $10 \mathrm{~kb}$ )

Additional file 3: Sets of membrane protein genes selected for phylogenetic inference. (XLSX $22 \mathrm{~kb}$ )

Additional file 4: Summary of unmapped reads taxonomic assignation by Centrifuge. (PNG $196 \mathrm{~kb}$ )

Additional file 5: Results of Xia's substitution saturation test using DAMBE. (DOCX $9 \mathrm{~kb}$ )

Additional file 6: Comparison of symbiont phylogenies inferred by gene set phylogeny and whole genome clustering. (PDF $307 \mathrm{~kb}$ )

Additional file 7 : Comparison of Buchnera aphidicola and mitochondrial phylogenies. (PDF $225 \mathrm{~kb}$ )

Additional file 8: Results of phylogenetic reconciliation by Jane. (DOCX $10 \mathrm{~kb}$ )

Additional file 9: Intra-host detection of distinct genotypes of $R$. insecticola. (DOCX $77 \mathrm{~kb})$

\section{Acknowledgements}

Authors warmly thank Jean Peccoud for advice on approaches to explore the evolutionary dynamics of pea aphid symbiont associations and the GenOuest Biolnformatics Platform that provided the computing resources necessary for bioinformatics analyses. We also thank the two anonymous referees for their helpful constructive comments that greatly contributed to improving this paper.

\section{Funding}

CG was supported by Université of Rennes 1 through a PhD grant. This work was supported by the Plant Health and Environment division of INRA and the ANR Speciaphid (ANR-11-BSV7-005-01) to JCS.

\section{Availability of data and materials}

Individual aphid sequencing datasets used for the current study are available under BioProject ID PRJNA255937. Pool sequencing datasets are available under BioProject IDs PRJNA385905 and PRJNA454786.

\section{Authors' contributions}

CG performed the analyses and wrote the paper, guided by $F L, E J, C M, C L$, and JCS. EJ supervised phylogenetic approaches and performed the reconciliation analyses. JCS led the writing of the paper. All authors read, revised, and approved the final manuscript.

\section{Ethics approval and consent to participate}

Not applicable.

\section{Consent for publication}

Not applicable.

\section{Competing interests}

The authors declare that they have no competing interests.

\section{Publisher's Note}

Springer Nature remains neutral with regard to jurisdictional claims in published maps and institutional affiliations.

\section{Author details}

'INRA, UMR 1349 INRA/Agrocampus Ouest/Université Rennes 1, Institut de Génétique, Environnement et Protection des Plantes (IGEPP), Le Rheu, France. ${ }^{2}$ Université Rennes 1, Inria, CNRS, IRISA, F-35000 Rennes, France.
${ }^{3}$ INRA, UMR CBGP (INRA/IRD/Cirad/Montpellier SupAgro), Campus International de Baillarguet, Montpellier, France.

Received: 25 May 2018 Accepted: 20 September 2018

Published online: 10 October 2018

\section{References}

1. Munson MA, Baumann P, Clark MA, Baumann L, Moran NA, Voegtlin DJ, et al. Evidence for the establishment of aphid-eubacterium endosymbiosis in an ancestor of four aphid families. J Bacteriol. 1991;173:6321-4 American Society for Microbiology. Available from: http://www.ncbi.nlm.nih.gov/ pubmed/1917864. Cited 26 Oct 2017.

2. Thao ML, Moran NA, Abbot P, Brennan EB, Burckhardt DH, Baumann P. Cospeciation of psyllids and their primary prokaryotic endosymbionts. Appl Environ Microbiol. 2000;66:2898-905 American Society for Microbiology. Available from: http://www.ncbi.nlm.nih.gov/pubmed/10877784. Cited 26 Oct 2017.

3. Gil R, Sabater-Muñoz B, Latorre A, Silva FJ, Moya A. Extreme genome reduction in Buchnera spp.: toward the minimal genome needed for symbiotic life. Proc Natl Acad Sci U S A. 2002;99:4454-8 National Academy of Sciences. Available from: http://www.pnas.org/content/99/7/4454. abstract?ijkey=69c46a4045e4960a41 f9952c500b9364a3f744e8\&keytype2=tf_ ipsecsha. Cited 15 Oct 2017.

4. Charlat S, Hurst GDD, Merçot H. Evolutionary consequences of Wolbachia infections. Trends Genet. 2003;19:217-23 Elsevier Current Trends. Available from: http://www.sciencedirect.com/science/article/pii/S0168952503000246. Cited 15 Oct 2017

5. Oliver KM, Russell JA, Moran NA, Hunter MS. Facultative bacterial symbionts in aphids confer resistance to parasitic wasps. Proc Natl Acad Sci. 2003;100: 1803-7 National Academy of Sciences. Available from: http://www.pnas.org/ cgi/doi/10.1073/pnas.0335320100. Cited 17 Oct 2017.

6. Russell JA, Moran NA. Costs and benefits of symbiont infection in aphids: variation among symbionts and across temperatures. Proc Biol Sci. 2006;273: 603-10 Available from: http://www.ncbi.n/m.nih.gov/pubmed/ 16537132\%5Cn http://www.pubmedcentral.nih.gov/articlerender.fcgi?artid= PMC1560055\%5Cn http://rspb.royalsocietypublishing.org.gate1.inist.fr/ content/273/1586/603.short. Cited 17 Oct 2017

7. Christian N, Whitaker BK, Clay K. Microbiomes: unifying animal and plant systems through the lens of community ecology theory. Front Microbiol. 2015;6:869 Frontiers. Available from: http://journal.frontiersin.org/Article/10. 3389/fmicb.2015.00869/abstract. Cited 18 Apr 2018.

8. McFall-Ngai M, Hadfield MG, Bosch TCG, Carey HV, Domazet-Lošo T, Douglas $A E$, et al. Animals in a bacterial world, a new imperative for the life sciences. Proc Natl Acad Sci. 2013;110:3229-36 National Academy of Sciences. Available from: http://www.pnas.org/lookup/doi/10.1073/pnas. 1218525110. Cited 18 Apr 2018

9. Rosenberg E, Koren O, Reshef L, Efrony R, Zilber-Rosenberg I. The role of microorganisms in coral health, disease and evolution. Nat Rev Microbiol. 2007;5:355-62 Available from: http://www.nature.com/doifinder/10.1038/ nrmicro1635.

10. Rohwer F, Seguritan V, Azam F, Knowlton N. Diversity and distribution of coral-associated bacteria. Mar Ecol Prog Ser. 2002;243:1-10.

11. Bordenstein SR, Theis KR. Host biology in light of the microbiome: ten principles of holobionts and hologenomes. PLoS Biol. 2015;13:e1002226 Waldor MK, editor. Cambridge University Press. Available from: http://dx plos.org/10.1371/journal.pbio.1002226. Cited 15 Oct 2017

12. Douglas $A E$, Werren $\mathrm{JH}$. Holes in the hologenome: why host-microbe symbioses are not holobionts. MBio. 2016;7:e02099 American Society for Microbiology. Available from: http://www.ncbi.nlm.nih.gov/pubmed/ 27034285. Cited 5 Oct 2017.

13. Douglas AE. How multi-partner endosymbioses function. Nat Rev Microbiol. 2016;14:731-43 Available from: http://www.nature.com/doifinder/10.1038/ nrmicro.2016.151. Cited 15 Nov 2017.

14. Jaspers E, Overmann J. Ecological significance of microdiversity: identical 165 rRNA gene sequences can be found in bacteria with highly divergent genomes and ecophysiologies. Appl Environ Microbiol. 2004;70:4831-9 American Society for Microbiology. Available from: http://www.ncbi.nlm.nih. gov/pubmed/15294821. Cited 5 Oct 2017.

15. Thomas GH, Zucker J, Macdonald SJ, Sorokin A, Goryanin I, Douglas AE. A fragile metabolic network adapted for cooperation in the symbiotic bacterium Buchnera aphidicola. BMC Syst Biol. 2009;3:24 BioMed Central. 
Available from: http://bmcsystbiol.biomedcentral.com/articles/10.1186/17520509-3-24. Cited 17 Nov 2017.

16. Albertsen M, Hugenholtz P, et al. AS-N, 2013 undefined. In: Albertsen, et al., editors. Genome sequences of rare, uncultured bacteria obtained by differential coverage binning of multiple metagenomes; 2013. researchgate. net. Available from: https://www.researchgate.net/profile/Per_Nielsen3/ publication/236939120_Genome_sequences_of_rare_uncultured_bacteria_ obtained_by_differential_coverage_binning_of_multiple_metagenomes/ links/0deec536d403ec8d7e000000.pdf. Cited 4 May 2018.

17. Sczyrba A, Hofmann P, Belmann P, Koslicki D, Janssen S, Dröge J, et al. Critical assessment of metagenome interpretation - a benchmark of metagenomics software. Nat Methods. 2017; Available from: http://www. nature.com/doifinder/10.1038/nmeth.4458.

18. Awad $S$, Irber $L$, Brown $C$. Evaluating metagenome assembly on a simple defined community with many strain variants. DoiOrg. 2017:155358 Available from: https:/ wuw.bionxiv.org/content/early/2017/07/03/155358. Cited 8 Nov 2017.

19. Segata N, Waldron L, Ballarini A, Narasimhan V, Jousson O, Huttenhower C. Metagenomic microbial community profiling using unique clade-specific marker genes. Nat Methods. 2012;9:811-4 Available from: http://www. pubmedcentral.nih.gov/articlerender.fcgi?artid=3443552\&tool= pmcentrez\&rendertype=abstract. Cited 15 Mar 2016.

20. Truong DT, Tett A, Pasolli E, Huttenhower C, Segata N. Microbial strain-level population structure \& genetic diversity from metagenomes. Genome Res. 2017;27:626-38 Cold Spring Harbor Laboratory Press. Available from: http:// www.ncbi.nlm.nih.gov/pubmed/28167665. Cited 4 May 2018.

21. Francis OE, Bendall M, Manimaran S, Hong C, Clement NL, Castro-Nallar E, et al. Pathoscope: species identification and strain attribution with unassembled sequencing data. Genome Res. 2013;23:1721-9 Available from: http://genome.cshlp.org/content/23/10/1721.abstract. Cited 9 Mar 2016.

22. Ahn TH, Chai J, Pan C. Sigma: strain-level inference of genomes from metagenomic analysis for biosurveillance. Bioinformatics. 2015;31:170-7 Oxford University Press. Available from: http://bioinformatics.oxfordjournals. org/content/early/2014/10/22/bioinformatics.btu641.full. Cited 9 Mar 2016.

23. Alneberg J, Bjarnason BS, de Bruijn I, Schirmer M, Quick J, ljaz UZ, et al. Binning metagenomic contigs by coverage and composition. Nat Methods. 2014;11:1144-6 Available from: http://www.ncbi.nlm.nih.gov/pubmed/ 25218180. Cited 8 Nov 2017.

24. Imelfort M, Parks D, Woodcroft BJ, Dennis P, Hugenholtz P, Tyson GW. GroopM: an automated tool for the recovery of population genomes from related metagenomes. PeerJ. 2014;2:e603 PeerJ Inc. Available from: https:// peerj.com/articles/603. Cited 30 Oct 2017.

25. Cleary B, Brito IL, Huang K, Gevers D, Shea T, Young S, et al. Detection of low-abundance bacterial strains in metagenomic datasets by eigengenome partitioning. Nat Biotechnol. 2015;33:1053-60 Available from: http://www.ncbi.nlm.nih.gov/pubmed/26368049\%5Cn http://www. pubmedcentral.nih.gov/articlerender.fcgi?artid=PMC4720164. Cited 4 July 2016.

26. Baumann P. Biology of bacteriocyte-associated endosymbionts of plant sapsucking insects. Annu Rev Microbiol. 2005;59:155-89 Annual Reviews. Available from: http://www.annualreviews.org/doi/10.1146/annurev.micro.59. 030804.121041. Cited 22 May 2018.

27. Tsuchida T, Koga R, Shibao H, Matsumoto T, Fukatsu T. Diversity and geographic distribution of secondary endosymbiotic bacteria in natural populations of the pea aphid, Acyrthosiphon pisum. Mol Ecol. 2002;11:212335 Blackwell Science Ltd. Available from: http://doi.wiley.com/10.1046/j. 1365-294X.2002.01606.x. Cited 12 Oct 2017.

28. Funk DJ, Wernegreen JJ, Moran NA. Intraspecific variation in symbiont genomes: bottlenecks and the aphid-Buchnera association. Genetics. 2001; 157:477-89 Available from: https://www.researchgate.net/profile/Nancy_ Moran/publication/12172609_Funk_D._J._Wernegreen_J_J._Moran_N._A._ Intraspecific_variation_in_symbiont_genomes_bottlenecks_and_the_AphidBuchnera_association._Genetics_157_477-489/links/ Odeec51f66815f0263000000. Cited 17 Aug 2017.

29. Sandström JP, Russell JA, White JP, Moran NA. Independent origins and horizontal transfer of bacterial symbionts of aphids. Mol Ecol. 2001;10:21728 Blackwell Science Ltd. Available from: http://doi.wiley.com/10.1046/j. 1365-294X.2001.01189.x. Cited 26 Sept 2017.

30. Oliver KM, Degnan PH, Burke GR, Moran NA. Facultative symbionts in aphids and the horizontal transfer of ecologically important traits. Annu Rev Entomol. 2010;55:247-66 Available from: http://www.annualreviews.org/doi/ 10.1146/annurev-ento-112408-085305. Cited 17 Oct 2017.
31. Simon J-C, Carre S, Boutin M, Prunier-Leterme N, Sabater-Munoz B, Latorre A, et al. Host-based divergence in populations of the pea aphid: insights from nuclear markers and the prevalence of facultative symbionts. Proc R Soc B Biol Sci. 2003;270:1703-12 Available from: http://rspb.royalsocietypublishing.org/ cgi/doi/10.1098/rspb.2003.2430. Cited 12 Oct 2017.

32. Peccoud J, Ollivier A, Plantegenest M, Simon J-C. A continuum of genetic divergence from sympatric host races to species in the pea aphid complex. Proc Natl Acad Sci U S A. 2009;106:7495-500 National Academy of Sciences. Available from: http://www.ncbi.nlm.nih.gov/pubmed/19380742\%5Cn http://www.pubmedcentral.nih.gov/articlerender.fcgi?artid=PMC2678636. Cited 20 July 2017.

33. Peccoud J, Simon J, Mclaughlin HJ, Moran NA. Post-Pleistocene radiation of the pea aphid complex revealed by rapidly evolving endosymbionts. Proc Natl Acad Sci U S A. 2009;106:16315-20 National Academy of Sciences. Available from: http://www.ncbi.nlm.nih.gov/pubmed/19805299. Cited 20 July 2017.

34. Gauthier JP, Outreman Y, Mieuzet L, Simon JC. Bacterial communities associated with host-adapted populations of pea aphids revealed by deep sequencing of 16S ribosomal DNA. PLoS One. 2015;10:e0120664 Duperron S, editor. Public Library of Science. Available from: http://dx.plos.org/10. 1371/journal.pone.0120664. Cited 21 Sept 2017.

35. Henry LM, Peccoud J, Simon JC, Hadfield JD, Maiden MJC, Ferrari J, et al. Horizontally transmitted symbionts and host colonization of ecological niches. Curr Biol. 2013;23:1713-7 Cell Press. Available from: http://www.sciencedirect. com/science/article/pii/S096098221300852X. Cited 14 Sept 2017.

36. Ferrari J, West JA, Via S, Godfray HCJ. Population genetic structure and secondary symbionts in host-associated populations of the pea aphid complex. Evolution ( $N$ Y). 2012;66:375-90 Blackwell Publishing Inc. Available from: http://doi.wiley.com/ 10.1111/j.1558-5646.2011.01436x. Cited 15 Nov 2017.

37. Leclair M, Pons I, Mahéo F, Morlière S, Simon JC, Outreman Y. Diversity in symbiont consortia in the pea aphid complex is associated with large phenotypic variation in the insect host. Evol Ecol. 2016;30:925-41 Springer International Publishing. Available from: http://link.springer.com/10.1007/ s10682-016-9856-1. Cited 24 July 2017.

38. Oliver KM, Moran N, Hunter MS. Variation in resistance to parasitism in aphids is due to symbionts not host genotype. Proc Natl Acad Sci U S A. 2005;102:12795-800 National Academy of Sciences. Available from: http:// www.pubmedcentral.nih.gov/articlerender.fcgi?artid=1200300\&tool= pmcentrez\&rendertype=abstract. Cited 15 Nov 2017.

39. Russell JA, Weldon S, Smith AH, Kim KL, Hu Y, Łukasik P, et al. Uncovering symbiont-driven genetic diversity across North American pea aphids. Mol Ecol. 2013;22:2045-59 Available from: http://doi.wiley.com/10.1111/mec. 12211. Cited 15 Nov 2017.

40. Peccoud J, Simon JC, Von Dohlen C, Coeur d'acier A, Plantegenest M, Vanlerberghe-Masutti F, et al. Evolutionary history of aphid-plant associations and their role in aphid diversification. Comptes Rendus - Biol. 2010;333:474-87 Elsevier Masson. Available from: http://www.sciencedirect. com/science/article/pii/S1631069110001095. Cited 17 Nov 2017.

41. Jaquiéry J, Stoeckel S, Nouhaud P, Mieuzet L, Mahéo F, Legeai F, et al. Genome scans reveal candidate regions involved in the adaptation to host plant in the pea aphid complex. Mol Ecol. 2012;21:5251-64 Wiley/Blackwell (10.1111). Available from: http://doi.wiley.com/10.1111/mec.12048. Cited 18 Apr 2018.

42. Gouin A, Legeai F, Nouhaud P, Whibley A, Simon JC, Lemaitre C. Wholegenome re-sequencing of non-model organisms: lessons from unmapped reads. Heredity (Edinb). 2015;1 14:494-501 Nature Publishing Group. Available from: http:/www.nature.com/hdy/journal/vaop/ncurrent/full/ hdy201485a.html. Cited 11 July 2017.

43. Li H, Durbin R. Fast and accurate short read alignment with BurrowsWheeler transform. Bioinformatics. 2009;25:1754-60 ACM. Available from: https://academic.oup.com/bioinformatics/article-lookup/doi/10.1093/ bioinformatics/btp324. Cited 14 Nov 2017.

44. Meseguer AS, Manzano-Marín A, Coeur d'Acier A, Clamens AL, Godefroid M, Jousselin E. Buchnera has changed flatmate but the repeated replacement of co-obligate symbionts is not associated with the ecological expansions of their aphid hosts. Mol Ecol. 2017;26:2363-78 Available from: http://doi. wiley.com/10.1111/mec.13910. Cited 13 Oct 2017.

45. Manzano-Marin A, Szabo G, Simon JC, Horn M, Latorre A. Happens in the best of subfamilies: establishment and repeated replacements of coobligate secondary endosymbionts within Lachninae aphids. Environ Microbiol. 2017;19:393-408 Wiley/Blackwell (10.1111). Available from: http:// doi.wiley.com/10.1111/1462-2920.13633. Cited 17 May 2018. 
46. Degnan PH, Moran NA. Diverse phage-encoded toxins in a protective insect endosymbiont. Appl Environ Microbiol. 2008;74:6782-91 American Society for Microbiology. Available from: http://www.ncbi.nlm.nih.gov/pubmed/ 18791000. Cited 21 Feb 2018.

47. Li H, Handsaker B, Wysoker A, Fennell T, Ruan J, Homer N, et al. The Sequence Alignment/Map format and SAMtools. Bioinformatics. 2009;25:2078-9 Oxford University Press. Available from: https://academic.oup.com/bioinformatics/ article-lookup/doi/10.1093/bioinformatics/btp352. Cited 24 Aug 2017.

48. Bankevich A, Nurk S, Antipov D, Gurevich AA, Dvorkin M, Kulikov AS, et al. SPAdes: a new genome assembly algorithm and its applications to single-cell sequencing. J Comput Biol. 2012;19:455-77 Mary Ann Liebert, Inc. 140 Huguenot Street, 3rd Floor New Rochelle, NY 10801 USA. Available from: http://online. liebertpub.com/doi/abs/10.1089/cmb.2012.0021. Cited 4 May 2018.

49. Rizk G, Gouin A, Chikhi R, Lemaitre C. MindTheGap: integrated detection and assembly of short and long insertions. Bioinformatics. 2014;30:3451-7 Oxford University Press. Available from: https://academic.oup.com/bioinformatics/ article-lookup/doi/10.1093/bioinformatics/btu545. Cited 21 Feb 2018.

50. Bolger AM, Lohse M, Usadel B. Trimmomatic: a flexible trimmer for Illumina sequence data. Bioinformatics. 2014:30:2114-20 Oxford University Press. Available from: https://academic.oup.com/bioinformatics/article-lookup/doi/ 10.1093/bioinformatics/btu170. Cited 24 Aug 2017

51. Kim D, Song L, Breitwieser FP, Salzberg SL. Centrifuge: rapid and sensitive classification of metagenomic sequences. Genome Res. 2016;26:1721-9 Cold Spring Harbor Laboratory Press. Available from: http://www.ncbi.nlm.nih. gov/pubmed/27852649. Cited 10 Nov 2017.

52. Breitwieser FP, Salzberg SL. Pavian: interactive analysis of metagenomics data for microbiomics and pathogen identification. bioRxiv. 2016:2014-7 Available from: http://biorxiv.org/content/early/2016/10/31/084715. Cited 10 Nov 2017.

53. Li H. A statistical framework for SNP calling, mutation discovery, association mapping and population genetical parameter estimation from sequencing data. Bioinformatics. 2011;27:2987-93 Oxford University Press. Available from: https://academic.oup.com/bioinformatics/article-lookup/doi/10.1093/ bioinformatics/btr509. Cited 4 May 2018.

54. Danecek P, Auton A, Abecasis G, Albers CA, Banks E, DePristo MA, et al. The variant call format and VCFtools. Bioinformatics. 2011;27:2156-8 Oxford University Press. Available from: https://academic.oup.com/bioinformatics/ article-lookup/doi/10.1093/bioinformatics/btr330. Cited 4 May 2018.

55. Petersen L, Bollback JP, Dimmic M, Hubisz M, Nielsen R. Genes under positive selection in Escherichia coli. Genome Res. 2007;17:1336-43 Cold Spring Harbor Laboratory Press. Available from: http://www.ncbi.n/m.nih. gov/pubmed/17675366. Cited 17 Apr 2018.

56. Katoh K, Kuma Kl, Toh H, Miyata T. MAFFT version 5: improvement in accuracy of multiple sequence alignment. Nucleic Acids Res. 2005;33:511-8 Oxford University Press. Available from: https://academic.oup.com/nar/ article-lookup/doi/10.1093/nar/gki198. Cited 30 Oct 2017.

57. Xia X, Xie Z. DAMBE: software package for data analysis in molecular biology and evolution. J Hered. 2001;92:371-3 Oxford University Press. Available from: https://academic.oup.com/jhered/article-lookup/doi/10.1093/ jhered/92.4.371. Cited 14 May 2018.

58. Stamatakis A, Ott M. Efficient computation of the phylogenetic likelihood function on multi-gene alignments and multi-core architectures. Philos Trans R Soc B Biol Sci. 2008;363:3977-84 Royal Society. Available from: http://rstb.royalsocietypublishing.org/cgi/doi/10.1098/rstb.2008.0163. Cited 30 Oct 2017.

59. Paradis E, Claude J, Strimmer K. APE: analyses of phylogenetics and evolution in R language. Bioinformatics. 2004;20:289-90 Oxford University Press. Available from: https://academic.oup.com/bioinformatics/articlelookup/doi/10.1093/bioinformatics/btg412. Cited 24 Aug 2017.

60. Galili T. Dendextend: an R package for visualizing, adjusting and comparing trees of hierarchical clustering. Bioinformatics. 2015;31:3718-20 Oxford University Press. Available from: https://academic.oup.com/bioinformatics/ article-lookup/doi/10.1093/bioinformatics/btv428. Cited 24 Aug 2017.

61. Gao X, Starmer J. Human population structure detection via multilocus genotype clustering. BMC Genet. 2007;8:34 BioMed Central. Available from: http:/ bmcgenet.biomedcentral.com/articles/10.1186/1471-2156-8-34. Cited 11 Jul 2017.

62. Conow C, Fielder D, Ovadia Y, Libeskind-Hadas R. Jane: a new tool for the cophylogeny reconstruction problem. Algorithms Mol Biol. 2010;5:16 BioMed Central. Available from: http://almob.biomedcentral.com/articles/10. 1186/1748-7188-5-16. Cited 30 Oct 2017.

63. Jousselin E, Desdevises $Y$, Coeur d'acier A. Fine-scale cospeciation between Brachycaudus and Buchnera aphidicola: bacterial genome helps define species and evolutionary relationships in aphids. Proc Biol Sci. 2009;276:18796 Available from: http://rspb.royalsocietypublishing.org/content/276/1654/ 187.short. Cited 30 Oct 2017.

64. Baldo L, Ayoub NA, Hayashi CY, Russell JA, Stahlhut JK, Werren JH. Insight into the routes of Wolbachia invasion: high levels of horizontal transfer in the spider genus Agelenopsis revealed by Wolbachia strain and mitochondrial DNA diversity. Mol Ecol. 2008;17:557-69 Blackwell Publishing Ltd. Available from: http://doi.wiley.com/10.1111/j.1365-294X.2007.03608.x. Cited 25 Sept 2017.

65. Baumann P, Baumann L, Lai CY, Rouhbakhsh D, Moran N, Clark M. Genetics, physiology, and evolutionary relationships of the genus Buchnera: intracellular symbionts of aphids. Annu Rev Microbiol. 1995;49:55-94 Available from: http://www.ncbi.nlm.nih.gov/pubmed/8561471. Cited 25 Sept 2017.

66. Moran NA, McLaughlin HJ, Sorek R. The dynamics and time scale of ongoing genomic erosion in symbiotic bacteria. Science (80- ). 2009;323: 379-82 Available from: http://www.sciencemag.org/cgi/doi/10.1126/science. 1167140. Cited 15 Nov 2017.

67. Chevignon G, Boyd BM, Brandt JW, Oliver KM, Strand MR. Culture-facilitated comparative genomics of the facultative symbiont hamiltonella defensa. Genome Biol Evol. 2018;10:786-802 Oxford University Press. Available from: https://academic.oup.com/gbe/article/10/3/786/4857210. Cited 4 May 2018.

68. Escobar-Zepeda A, De León AVP, Sanchez-Flores A. The road to metagenomics: from microbiology to DNA sequencing technologies and bioinformatics. Front Genet. 2015;6:348 Frontiers. Available from: http:// journal.frontiersin.org/Article/10.3389/fgene.2015.00348/abstract. Cited 15 June 2016.

69. Wang Z, Su XM, Wen J, Jiang LY, Qiao GX. Widespread infection and diverse infection patterns of Wolbachia in Chinese aphids. Insect Sci. 2014;21:31325 Wiley/Blackwell (10.1111). Available from: http://doi.wiley.com/10.1111/ 1744-7917.12102. Cited 15 May 2018.

70. Russell JA, Weldon S, Smith AH, Kim KL, Hu Y, Łukasik P, et al. Uncovering symbiont-driven genetic diversity across North American pea aphids. Mol Ecol. 2013;22:2045-59 Available from: http://www.ncbi.nlm.nih.gov/ pubmed/23379399. Cited 15 May 2018.

71. François S, Filloux D, Fernandez E, Ogliastro M, Roumagnac P. Viral metagenomics approaches for high-resolution screening of multiplexed arthropod and plant viral communities. Methods Mol Biol. 2018;1746:77-95 Humana Press, New York, NY. Available from: http://link.springer.com/10. 1007/978-1-4939-7683-6_7. Cited 15 May 2018.

72. Colman DR, Toolson EC, Takacs-Vesbach CD. Do diet and taxonomy influence insect gut bacterial communities? Mol Ecol. 2012;21:5124-37 Available from: http://doi.wiley.com/10.1111/j.1365-294X.2012.05752.x. Cited 30 Oct 2017.

73. Jing X, Wong ACN, Chaston JM, Colvin J, McKenzie CL, Douglas AE. The bacterial communities in plant phloem-sap-feeding insects. Mol Ecol. 2014; 23:1433-44 Available from: http://doi.wiley.com/10.1111/mec.12637. Cited 30 Oct 2017.

74. Moran NA, Munson MA, Baumann P, Ishikawa H. A molecular clock in endosymbiotic bacteria is calibrated using the insect hosts. Proc R Soc B Biol Sci. 1993;253:167-71 Available from: http://rspb.royalsocietypublishing. org/cgi/doi/10.1098/rspb.1993.0098. Cited 26 Sept 2017.

75. Moran NA, Yun Y. Experimental replacement of an obligate insect symbiont. Proc Natl Acad Sci. 2015;112:2093-6 National Academy of Sciences. Available from: http://www.pnas.org/lookup/doi/10.1073/pnas.1420037112. Cited 17 Nov 2017.

76. Moran NA, von Dohlen CD, Baumann P. Faster evolutionary rates in endosymbiotic bacteria than in cospeciating insect hosts. J Mol Evol. 1995; 41:727-31 Springer-Verlag. Available from: http://link.springer.com/10.1007/ BF00173152. Cited 13 Oct 2017.

77. Chong RA, Moran NA. Evolutionary loss and replacement of Buchnera, the obligate endosymbiont of aphids. ISME J. 2018;12:898-908 Nature Publishing Group. Available from: http://www.nature.com/articles/s41396017-0024-6. Cited 11 May 2018.

78. Rock DI, Smith AH, Joffe J, Albertus A, Wong N, O'Connor M, et al. Contextdependent vertical transmission shapes strong endosymbiont community structure in the pea aphid, Acyrthosiphon pisum. Mol Ecol. 2017.

79. Moran NA, McCutcheon JP, Nakabachi A. Genomics and evolution of heritable bacterial symbionts. Annu Rev Genet. 2008;42:165-90 Available from: http://www.annualreviews.org/doi/10.1146/annurev.genet.41.110306. 130119. Cited 17 Nov 2017. 
80. McCutcheon JP, Moran NA. Extreme genome reduction in symbiotic bacteria. Nat Rev Microbiol. 2011;10:nrmicro2670 Nature Publishing Group. Available from: http://www.nature.com/doifinder/10.1038/nrmicro2670. Cited 17 Nov 2017.

81. Łukasik P, Guo H, van Asch M, Henry LM, Godfray HCJ, Ferrari J. Horizontal transfer of facultative endosymbionts is limited by host relatedness. Evolution (N Y). 2015;69:2757-66.

82. Henry LM, Maiden MCJ, Ferrari J, Godfray HCJ. Insect life history and the evolution of bacterial mutualism. Ecol Lett. 2015;18:516-25 Bourke A, editor. Wiley/Blackwell (10.1111). Available from: http://doi.wiley.com/10.1111/ele. 12425. Cited 24 May 2018.

83. Valette V, Bitome Essono PY, Le Clec'h W, Johnson M, Bech N, Grandjean F. Multi-infections of feminizing Wolbachia strains in natural populations of the terrestrial isopod Armadillidium vulgare. PLoS One. 2013;8:e82633 Moreira LA, editor. Public Library of Science. Available from: http://dx.plos. org/10.1371/journal.pone.0082633. Cited 27 Sept 2017.

84. Brandt JW, Chevignon G, Oliver KM, Strand MR. Culture of an aphid heritable symbiont demonstrates its direct role in defence against parasitoids. Proc R Soc B Biol Sci. 2017;284:20171925 The Royal Society. Available from: http://rspb.royalsocietypublishing.org/lookup/doi/10.1098/ rspb.2017.1925. Cited 17 May 2018.

85. Richards S, Gibbs RA, Gerardo NM, Moran N, Nakabachi A, Stern D, et al. Genome sequence of the pea aphid Acyrthosiphon pisum. PLoS Biol. 2010;8: e1000313 Eisen JA, editor. Elsevier Academic Press. Available from: http://dx. plos.org/10.1371/journal.pbio.1000313. Cited 21 Sept 2017.

86. Shigenobu S, Watanabe H, Hattori M, Sakaki Y, Ishikawa H. Genome sequence of the endocellular bacterial symbiont of aphids Buchnera sp. APS. Nature. 2000;407:81-6 Available from: http://www.ncbi.nlm.nih.gov/ pubmed/10993077. Cited 8 Aug 2017.

87. Degnan PH, Yu Y, Sisneros N, Wing R, Moran N. Hamiltonella defensa, genome evolution of protective bacterial endosymbiont from pathogenic ancestors. Proc Natl Acad Sci U S A. 2009;106:9063-8 Available from: http:// www.pubmedcentral.nih.gov/articlerender.fcgi?artid=2690004\&tool= pmcentrez\&rendertype=abstract. Cited 8 Aug 2017.

88. van der Wilk F, Dullemans AM, Verbeek M, van den Heuvel JFJ. Isolation and characterization of APSE-1, a bacteriophage infecting the secondary endosymbiont of Acyrthosiphon pisum. Virology. 1999;262:104-13 Available from: http://www.sciencedirect.com/science/article/pii/ S0042682299999026\%5Cn http://www.sciencedirect.com/science/article/pii/ S0042682299999026/pdf?md5=762e5637961a08d60392280c87f3da1e\&pid= 1-s2.0-S0042682299999026-main.pdf. Cited 8 Aug 2017.

89. Hansen AK, Vorburger C, Moran NA. Genomic basis of endosymbiont-conferred protection against an insect parasitoid. Genome Res. 2012;22:106-14 Available from: http://www.ncbi.nIm.nih.gov/pubmed/21948522. Cited 8 Aug 2017.

90. Degnan PH, Leonardo TE, Cass BN, Hurwitz B, Stern D, Gibbs RA, et al. Dynamics of genome evolution in facultative symbionts of aphids. Environ Microbiol. 2010;12:2060-9 Blackwell Publishing Ltd. Available from: http:// doi.wiley.com/10.1111/j.1462-2920.2009.02085.x. Cited 8 Aug 2017.

91. Burke GR, Moran NA. Massive genomic decay in Serratia symbiotica, a recently evolved symbiont of aphids. Genome Biol Evol. 2011;3:195-208 Available from: http://www.ncbi.n/m.nih.gov/pubmed/21266540. Cited 8 Aug 2017.

92. Nikoh N, Tsuchida T, Maeda T, Yamaguchi K, Shigenobu S, Koga R, et al. Genomic insight into symbiosis-induced insect color change by a facultative bacterial endosymbiont, "Candidatus Rickettsiella viridis". MBio. 2018;9:e00890-18 American Society for Microbiology. Available from: http:// www.ncbi.nlm.nih.gov/pubmed/29895637. Cited 7 Aug 2018.

93. Klasson L, Westberg J, Sapountzis P, Naslund K, Lutnaes Y, Darby AC, et al. The mosaic genome structure of the Wolbachia wRi strain infecting Drosophila simulans. Proc Natl Acad Sci. 2009;106:5725-30 National Academy of Sciences. Available from: http://www.pnas.org/cgi/doi/10.1073/ pnas.0810753106. Cited 7 Aug 2018

Ready to submit your research? Choose BMC and benefit from:

- fast, convenient online submission

- thorough peer review by experienced researchers in your field

- rapid publication on acceptance

- support for research data, including large and complex data types

- gold Open Access which fosters wider collaboration and increased citations

- maximum visibility for your research: over $100 \mathrm{M}$ website views per year

At BMC, research is always in progress.

Learn more biomedcentral.com/submissions 